\title{
Lattices in almost abelian Lie groups with locally conformal Kähler or symplectic structures
}

\section{A. Andrada \& M. Origlia}

\section{manuscripta mathematica}

ISSN 0025-2611

manuscripta math.

DOI 10.1007/s00229-017-0938-3

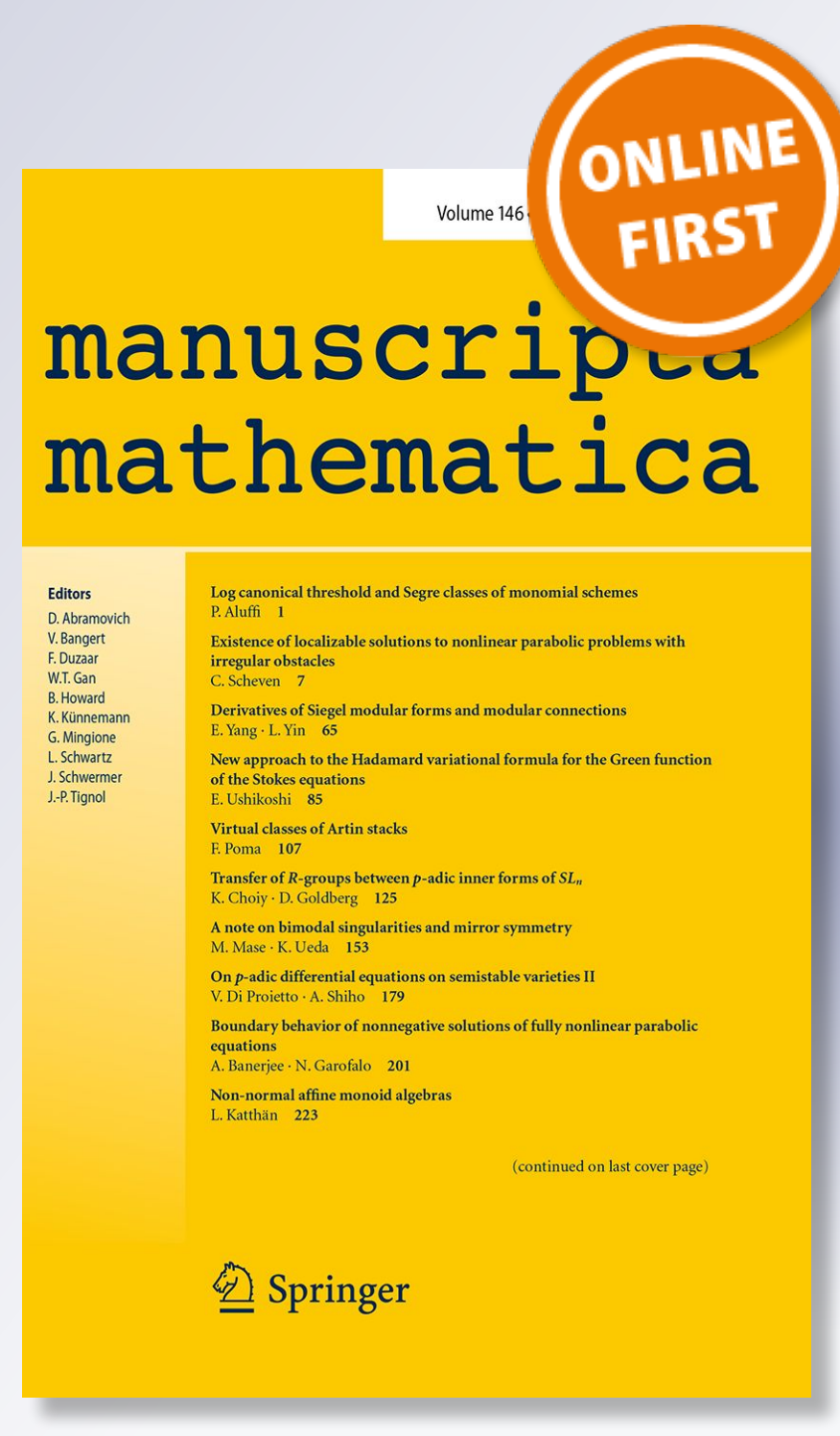

照 Springer 
Your article is protected by copyright and all rights are held exclusively by SpringerVerlag Berlin Heidelberg. This e-offprint is for personal use only and shall not be selfarchived in electronic repositories. If you wish to self-archive your article, please use the accepted manuscript version for posting on your own website. You may further deposit the accepted manuscript version in any repository, provided it is only made publicly available 12 months after official publication or later and provided acknowledgement is given to the original source of publication and a link is inserted to the published article on Springer's website. The link must be accompanied by the following text: "The final publication is available at link.springer.com". 
A. Andrada $\cdot$ M. Origlia

\title{
Lattices in almost abelian Lie groups with locally conformal Kähler or symplectic structures
}

\author{
Received: 14 November 2016/ Accepted: 13 April 2017
}

\begin{abstract}
We study the existence of lattices in almost abelian Lie groups that admit left invariant locally conformal Kähler or locally conformal symplectic structures in order to obtain compact solvmanifolds equipped with these geometric structures. In the former case, we show that such lattices exist only in dimension 4 , while in the latter case we provide examples of such Lie groups admitting lattices in any even dimension.
\end{abstract}

\section{Introduction}

The aim of this article is to study the existence of lattices in a family of solvable Lie groups equipped with certain left invariant geometric structures, namely, locally conformal Kähler structures and locally conformal symplectic structures.

We recall that a $2 n$-dimensional Hermitian manifold $(M, J, g)$ is called locally conformal Kähler (LCK for short) if $g$ can be rescaled locally, in a neighborhood of any point in $M$, so as to be Kähler. If $\omega$ denotes the fundamental 2-form of $(J, g)$ defined by $\omega(X, Y)=g(J X, Y)$ for any $X, Y$ vector fields on $M$, it is well known that the LCK condition is equivalent to the existence of a closed 1-form $\theta$ on $M$ such that $d \omega=\theta \wedge \omega$. This 1 -form $\theta$ is called the Lee form. These manifolds are a natural generalization of Kähler manifolds, and they have been much studied by many authors since the work of I. Vaisman in the ' 70 s (see for instance $[11,12,33$ 35,42]).

A generalization of this class of manifolds is given by the so-called locally conformal symplectic (LCS) manifolds, i.e., those manifolds carrying a non-degenerate 2-form $\omega$ satisfying $d \omega=\theta \wedge \omega$ for some closed 1-form $\theta$. Locally conformal symplectic manifolds were considered by Lee in [27] and they have been firstly studied by Vaisman in [43]. Some recent results can be found in $[5,16,17,26]$, among others.

Any left invariant LCK or LCS structure on a simply connected Lie group $G$ gives rise naturally to an LCK or LCS structure on a quotient $\Gamma \backslash G$ of $G$ by a discrete subgroup. In this article we will consider solvable Lie groups $G$ and discrete subgroups $\Gamma$ such that $\Gamma \backslash G$ is compact, i.e., $\Gamma$ is a lattice in $G$; the compact quotient

A. Andrada · M. Origlia ( $\varangle$ ): FaMAF-CIEM, Universidad Nacional de Córdoba, Ciudad Universitaria, 5000 Córdoba, Argentina e-mail: origlia@famaf.unc.edu.ar

A. Andrada: e-mail: andrada@famaf.unc.edu.ar

Mathematics Subject Classification: 22E25 - 22E40 $\cdot$ 53C15 $\cdot$ 53C55 $\cdot$ 53D05 
is called a solvmanifold. In general, it is difficult to determine whether a given Lie group admits lattices. However, there are two classes of Lie groups for which a criterion exists: for nilpotent Lie groups, there is the well known Malcev's theorem [29], while for almost abelian Lie groups there is a characterization by Bock ([7], see Proposition 2.3 below). We recall that a Lie group is called almost abelian when its Lie algebra has an abelian ideal of codimension one. Left invariant LCK or LCS structures on nilpotent Lie groups have been thoroughly studied in $[6,39]$, therefore we will focus on the class of almost abelian Lie groups. This class has appeared recently in several different contexts (see for instance [4, 7,9, 24,25]).

LCK and LCS structures on Lie groups and Lie algebras (and also in their compact quotients by discrete subgroups, if they exist) have been considered by several authors lately. For instance, it was shown in [39] that an LCK nilpotent Lie algebra is isomorphic to $\mathfrak{h}_{2 n+1} \times \mathbb{R}$, where $\mathfrak{h}_{2 n+1}$ denotes the $(2 n+1)$-dimensional Heisenberg Lie algebra. In [22] it is proved that some solvmanifolds with left invariant complex structures do not admit Vaisman metrics, i.e. LCK metrics with parallel Lee form. Moreover, it is shown that some Oeljeklaus-Toma manifolds (used to disprove a conjecture by I. Vaisman) are in fact solvmanifolds with invariant LCK structures. In [1] it is proved that any reductive Lie group admitting left invariant LCK metrics is locally isomorphic to $U(2)$ and $G L(2, \mathbb{R})$. In [3] the authors prove that if a solvmanifold with an abelian complex structure (i.e. $[J x, J y]=[x, y]$ for any $x, y$ in the corresponding Lie algebra) admits an invariant LCK metric then the Lie algebra is isomorphic to $\mathfrak{h}_{2 n+1} \times \mathbb{R}$. In [6] a structure theorem for Lie algebras admitting LCS structures of the first kind is given, and 6-dimensional LCS nilpotent Lie algebras are classified.

The outline of this article is as follows. In Sect. 2 we review some known results about LCK and LCS structures and almost abelian Lie groups.

In Sect. 3 we characterize the Lie algebras of the almost abelian Lie groups that admit a left invariant LCK structure (Theorem 3.3) in any even dimension $\geq 4$. Moreover, we determine whether these Lie groups admit lattices, proving that this happens only in dimension 4 (Theorem 3.7), using the criterion for the existence of lattices in almost abelian Lie groups given in [7]. In particular, there is a one-parameter family of unimodular almost abelian 4-dimensional Lie groups with left invariant LCK structures, with countably many of them admitting lattices (Theorem 3.9). The 4-dimensional solvmanifolds thus obtained are Inoue surfaces of type $S^{0}$ (see [18]).

In Sect. 4 we study LCS structures on almost abelian Lie algebras and we get a characterization of these Lie algebras in any even dimension (see Theorem 4.1 for dimension 6 or higher and Theorem 4.5 for dimension 4). Furthermore, we establish which of these LCS structures are of the first kind or of the second kind. We also determine whether the 4-dimensional Lie groups associated to these Lie algebras admit lattices (Theorem 4.8) and finally, we build in each dimension greater than or equal to 6 a family of almost abelian solvmanifolds admitting an LCS structure of the second kind which carry no invariant complex structure. Moreover, we determine the Betti numbers associated to the de Rham cohomology and the adapted (or Lichnerowicz) cohomology of these solvmanifolds in low dimensions. 


\section{Preliminaries}

\subsection{Locally conformal structures on manifolds}

Let $(M, J, g)$ be a $2 n$-dimensional Hermitian manifold, where $J$ is a complex structure and $g$ is a Hermitian metric. $(M, J, g)$ is a locally conformal Kähler manifold (LCK for short) if there exists an open covering $\left\{U_{i}\right\}_{i \in I}$ of $M$ and a family $\left\{f_{i}\right\}_{i \in I}$ of $C^{\infty}$ functions, $f_{i}: U_{i} \rightarrow \mathbb{R}$, such that each local metric

$$
g_{i}=\left.\exp \left(-f_{i}\right) g\right|_{U_{i}}
$$

is Kähler. Also $(M, J, g)$ is globally conformal Kähler $(\mathrm{GCK})$ if there exists a $C^{\infty}$ function, $f: M \rightarrow \mathbb{R}$, such that the metric $\exp (-f) g$ is Kähler.

An equivalent characterization of an LCK manifold can be given in terms of the fundamental form $\omega$, which is defined by $\omega(X, Y)=g(J X, Y)$, for all $X, Y \in$ $\mathfrak{X}(M)$. Indeed, a Hermitian manifold $(M, J, g)$ is LCK if and only if there exists a closed 1-form $\theta$ globally defined on $M$ such that

$$
d \omega=\theta \wedge \omega .
$$

This closed 1-form $\theta$ is called the Lee form (see [27]). The Lee form is completely determined by $\omega$, and there is an explicit formula given by

$$
\theta=-\frac{1}{n-1}(\delta \omega) \circ J
$$

where $\delta$ is the codifferential and $2 n$ is the dimension of $M$.

It can be seen that $(M, J, g)$ is LCK if and only if

$$
\left(\nabla_{X} J\right) Y=\frac{1}{2}\left\{\theta(J Y) X-\theta(Y) J X+g(X, Y) J \theta^{\#}+\omega(X, Y) \theta^{\#}\right\},
$$

for all $X, Y \in \mathfrak{X}(M)$, where $\theta^{\#}$ is the dual vector field of the 1 -form $\theta$ and $\nabla$ is the Levi-Civita connection associated to $g$. This shows that LCK manifolds belong to the class $\mathcal{W}_{4}$ of the Gray-Hervella classification of almost Hermitian manifolds [14].

A generalization of an LCK structure is given by a locally conformal symplectic structure (LCS for short), that is, a non degenerate 2 -form $\omega$ on the manifold $M$ such that there exists an open cover $\left\{U_{i}\right\}$ and smooth functions $f_{i}$ on $U_{i}$ such that

$$
\omega_{i}=\exp \left(-f_{i}\right) \omega
$$

is a symplectic form on $U_{i}$. This condition is equivalent to requiring that

$$
d \omega=\theta \wedge \omega
$$

for some closed 1-form $\theta$, called again the Lee form. Moreover, $M$ is called globally conformal symplectic (GCS) if there exist a $C^{\infty}$ function, $f: M \rightarrow \mathbb{R}$, such that $\exp (-f) g$ is a symplectic form. Equivalently, $M$ is a GCS manifold if there exists a exact 1-form $\theta$ globally defined on $M$ such that $d \omega=\theta \wedge \omega$.

We note that in the LCS case the Lee form is also uniquely determined by the non degenerate 2-form $\omega$, but there is not an explicit formula for $\theta$ such as (3) in the LCK case. The pair $(\omega, \theta)$ will be called an LCS structure on $M$. 
It is well known that

- If $(\omega, \theta)$ is an LCS structure on $M$, then $\omega$ is symplectic if and only if $\theta=0$. Indeed, $\theta \wedge \omega=0$ and $\omega$ non degenerate imply $\theta=0$. Accordingly, an LCK structure $(J, g)$ is Kähler if and only if $\theta=0$.

- If $\omega$ is a non degenerate 2-form on $M$, with $\operatorname{dim} M \geq 6$, such that (2) holds for some 1 -form $\theta$ then $\theta$ is automatically closed and therefore $M$ is LCS.

We recall next a definition due to Vaisman ([43]). If $(\omega, \theta)$ is an LCS structure on $M$, a vector field $X$ is called an infinitesimal automorphism of $(\omega, \theta)$ if $\mathrm{L}_{X} \omega=$ 0 , where $\mathrm{L}$ denotes the Lie derivative. This implies $\mathrm{L}_{X} \theta=0$ as well and, as a consequence, $\theta(X)$ is a constant function on $M$. If there exists an infinitesimal automorphism $X$ such that $\theta(X) \neq 0$, the LCS structure $(\omega, \theta)$ is said to be of the first kind, and it is of the second kind otherwise.

Since $\theta$ is closed, we can deform the de Rham differential $d$ to obtain the adapted differential operator

$$
d_{\theta} \alpha=d \alpha-\theta \wedge \alpha
$$

This operator satisfies $d_{\theta}^{2}=0$, thus it defines the adapted cohomology $H_{\theta}^{*}(M)$ of $M$ relative to the closed 1-form $\theta$, also called the Lichnerowicz cohomology. It is known that if $M$ is a compact oriented $n$-dimensional manifold, then $H_{\theta}^{0}(M)=$ $H_{\theta}^{n}(M)=0$ for any non exact closed 1 -form $\theta$ (see for instance [15,16]). For any LCS structure $(\omega, \theta)$ on $M$, the 2-form $\omega$ defines a cohomology class $[\omega]_{\theta} \in$ $H_{\theta}^{2}(M)$, since $d_{\theta} \omega=d \omega-\theta \wedge \omega=0$. It was proved in [43] that if the LCS structure is of the first kind then $\omega$ is $d_{\theta}$-exact, i.e. $[\omega]_{\theta}=0$.

\subsection{Left invariant LCK and LCS structures on Lie groups and its compact quotients}

Let $G$ be a Lie group with a left invariant Hermitian structure $(J, g)$. If $(J, g)$ satisfies the LCK condition (2), then $(J, g)$ is called a left invariant LCK structure on the Lie group $G$. Clearly, the fundamental 2-form is left invariant and, using (3), it is easy to see that the corresponding Lee form $\theta$ on $G$ is also left invariant.

This fact allows us to define LCK structures on Lie algebras. We recall that a complex structure $J$ on a Lie algebra $\mathfrak{g}$ is an endomorphism $J: \mathfrak{g} \rightarrow \mathfrak{g}$ satisfying $J^{2}=-$ Id and

$$
N_{J}=0, \quad \text { where } N_{J}(x, y)=[J x, J y]-[x, y]-J([J x, y]+[x, J y]),
$$

for any $x, y \in \mathfrak{g}$.

Let $\mathfrak{g}$ be a Lie algebra, $J$ a complex structure and $\langle\cdot, \cdot\rangle$ a Hermitian inner product on $\mathfrak{g}$, with $\omega \in \bigwedge^{2} \mathfrak{g}^{*}$ the fundamental 2-form. We say that $(\mathfrak{g}, J,\langle\cdot, \cdot\rangle)$ is locally conformal Kähler (LCK) if there exists $\theta \in \mathfrak{g}^{*}$, with $d \theta=0$, such that

$$
d \omega=\theta \wedge \omega .
$$

In the same fashion, an LCS structure $(\omega, \theta)$ on a Lie group $G$ is called left invariant if $\omega$ is left invariant, and this easily implies that $\theta$ is also left invariant. 
Accordingly, we say that a Lie algebra $\mathfrak{g}$ admits a locally conformal symplectic (LCS) structure if there exist $\omega \in \bigwedge^{2} \mathfrak{g}^{*}$ and $\theta \in \mathfrak{g}^{*}$, with $\omega$ non degenerate and $\theta$ closed, such that (4) is satisfied.

As in the case of manifolds we have that an LCS structure $(\omega, \theta)$ on a Lie algebra $\mathfrak{g}$ can be of the first kind or of the second kind. Indeed, let us denote by $\mathfrak{g}_{\omega}$ the set of infinitesimal automorphisms of the LCS structure, that is,

$$
\mathfrak{g}_{\omega}=\left\{x \in \mathfrak{g}: \mathrm{L}_{x} \omega=0\right\}=\{x \in \mathfrak{g}: \omega([x, y], z)+\omega(y,[x, z])=0 \text { for all } y, z \in \mathfrak{g}\} .
$$

Note that $\mathfrak{g}_{\omega} \subset \mathfrak{g}$ is a Lie subalgebra, thus the restriction of $\theta$ to $\mathfrak{g}_{\omega}$ is a Lie algebra morphism called Lee morphism. The LCS structure $(\omega, \theta)$ is said to be of the first kind if the Lee morphism is surjective, and of the second kind if it is identically zero (see [6]).

For a Lie algebra $\mathfrak{g}$ and a closed 1 -form $\theta \in \mathfrak{g}^{*}$ we also have the adapted cohomology $H_{\theta}^{*}(\mathfrak{g})$ defined by the differential operator

$$
d_{\theta} \alpha=d \alpha-\theta \wedge \alpha
$$

on $\bigwedge^{*} \mathfrak{g}^{*}$. According to [31], this adapted cohomology coincides with the Lie algebra cohomology of $\mathfrak{g}$ with coefficients in a 1-dimensional $\mathfrak{g}$-module $V_{\theta}$, where the action of $\mathfrak{g}$ on $V_{\theta}$ is given by

$$
X v=-\theta(X) v, \quad X \in \mathfrak{g}, v \in V_{\theta} .
$$

The fact that $\theta$ is closed guarantees that this is a Lie algebra representation.

If the Lie group is simply connected then any left invariant LCK or LCS structure turns out to be globally conformal to a Kähler or symplectic structure. Therefore we will study compact quotients of such a Lie group by discrete subgroups, which will be non simply connected and will inherit an LCK or LCS structure. Recall that a discrete subgroup $\Gamma$ of a simply connected Lie group $G$ is called a lattice if the quotient $\Gamma \backslash G$ is compact. The quotient $\Gamma \backslash G$ is known as a solvmanifold if $G$ is solvable and as a nilmanifold if $G$ is nilpotent, and in these cases we have that $\pi_{1}(\Gamma \backslash G) \cong \Gamma$. We note that an LCS structure of the first kind on a Lie algebra induces an LCS structure of the first kind on any compact quotient of the corresponding simply connected Lie group by a discrete subgroup.

In the case when $G$ is completely solvable, i.e. it is a solvable Lie group such that the endomorphisms $\operatorname{ad}_{X}$ of its Lie algebra $\mathfrak{g}$ have only real eigenvalues for all $X \in \mathfrak{g}$, the de Rham and adapted cohomology of $\Gamma \backslash G$ can be computed in terms of the cohomology of $\mathfrak{g}$. Indeed, Hattori proved in [19] that if $V$ is a finite dimensional triangular ${ }^{1} \mathfrak{g}$-module, then $\bar{V}:=C^{\infty}(\Gamma \backslash G) \otimes V$ is a $\mathfrak{X}(\Gamma \backslash G)$-module and there is an isomorphism

$$
H^{*}(\mathfrak{g}, V) \cong H^{*}(\mathfrak{X}(\Gamma \backslash G), \bar{V}) .
$$

Therefore:

${ }^{1} \mathrm{~A} \mathfrak{g}$-module $V$ is called triangular if the endomorphisms of $V$ defined by $v \mapsto X v$ have only real eigenvalues for any $X \in \mathfrak{g}$. 
- If $V=\mathbb{R}$ is the trivial $\mathfrak{g}$-module, then the right-hand side in (7) gives the usual de Rham cohomology of $\Gamma \backslash G$, so that

$$
H^{*}(\mathfrak{g}) \cong H_{d R}^{*}(\Gamma \backslash G)
$$

- If $V=V_{\theta}$ with the action given by (6), then we can identify $\bar{V}$ with $C^{\infty}(\Gamma \backslash G)$ and the action of $\mathfrak{X}(\Gamma \backslash G)$ on $C^{\infty}(\Gamma \backslash G)$ is given by

$$
X \cdot f=X f-\theta(X) f, \quad X \in \mathfrak{g}, f \in C^{\infty}(\Gamma \backslash G) .
$$

Here we are using that there is a natural inclusion $\mathfrak{g} \hookrightarrow \mathfrak{X}(\Gamma \backslash G)$ and a bijection $C^{\infty}(\Gamma \backslash G) \otimes \mathfrak{g} \rightarrow \mathfrak{X}(\Gamma \backslash G)$ given by $f \otimes X \mapsto f X$. As a consequence, in this case (7) becomes (cf. [31, Corollary 4.1])

$$
H_{\theta}^{*}(\mathfrak{g}) \cong H_{\theta}^{*}(\Gamma \backslash G)
$$

In particular, $H_{d R}^{*}(\Gamma \backslash G)$ and $H_{\theta}^{*}(\Gamma \backslash G)$ do not depend on the lattice $\Gamma$.

\subsection{Almost abelian Lie groups}

In this article we will focus on a family of solvable Lie groups, namely, the almost abelian ones. We recall that a Lie group $G$ is said to be almost abelian if its Lie algebra $\mathfrak{g}$ has a codimension one abelian ideal. Such a Lie algebra will be called almost abelian, and it can be written as $\mathfrak{g}=\mathbb{R} f_{1} \ltimes_{\mathrm{ad}_{f_{1}}} \mathfrak{u}$, where $\mathfrak{u}$ is an abelian ideal of $\mathfrak{g}$, and $\mathbb{R}$ is generated by $f_{1}$. Accordingly, the Lie group $G$ is a semidirect product $G=\mathbb{R} \ltimes_{\phi} \mathbb{R}^{d}$ for some $d \in \mathbb{N}$, where the action is given by $\phi(t)=e^{t \operatorname{ad}_{f_{1}}}$. We point out that an almost abelian Lie algebra is nilpotent if and only if the operator $\left.\operatorname{ad}_{f_{1}}\right|_{\mathfrak{u}}$ is nilpotent.

Regarding the isomorphism classes of almost abelian Lie algebras, it can be proved that

Lemma 2.1. Two almost abelian Lie algebras $\mathfrak{g}_{1}=\mathbb{R} f_{1} \ltimes_{\mathrm{ad}_{f_{1}}} \mathfrak{u}_{1}$ and $\mathfrak{g}_{2}=$ $\mathbb{R} f_{2} \ltimes_{\operatorname{ad}_{f_{2}}} \mathfrak{u}_{2}$ are isomorphic if and only if there exists $c \neq 0$ such that $\operatorname{ad}_{f_{1}}$ and $c$ ad $_{f_{2}}$ are conjugate.

See [2] for a proof in the case $\operatorname{dim} \mathfrak{g}=4$.

Remark 2.2. Note that a codimension one abelian ideal of an almost abelian Lie algebra is almost always unique. Indeed, if $\mathfrak{u}$ and $\mathfrak{v}$ are codimension one abelian ideals of $\mathfrak{g}$, with $\mathfrak{u} \neq \mathfrak{v}$, then $\mathfrak{u} \cap \mathfrak{v}$ is a codimension two abelian ideal, and we can decompose $\mathfrak{g}=\mathbb{R} u_{0} \oplus \mathbb{R} v_{0} \oplus \mathfrak{u} \cap \mathfrak{v}$ for some $u_{0} \in \mathfrak{u}-\mathfrak{v}, v_{0} \in \mathfrak{v}-\mathfrak{u}$. If $\left[u_{0}, v_{0}\right]=0$, then $\mathfrak{g}$ is abelian, and if $\left[u_{0}, v_{0}\right] \neq 0$, then $\left[u_{0}, v_{0}\right]$ generates the commutator ideal of $\mathfrak{g}$, and therefore $\mathfrak{g}$ is isomorphic to $\mathfrak{h}_{3} \times \mathbb{R}^{s}$ for some $s \geq 0$, where $\mathfrak{h}_{3}$ denotes the 3-dimensional Heisenberg Lie algebra.

As a consequence, in any other case the codimension one abelian ideal is unique. 
An important feature concerning almost abelian Lie groups is that there exists a criterion to determine when such a Lie group admits lattices. In general, it is not easy to determine if a given Lie group $G$ admits a lattice. A well known restriction is that if this is the case then $G$ must be unimodular ([32]), i.e. the Haar measure on $G$ is left and right invariant, or equivalently, when $G$ is connected, $\operatorname{tr}\left(\operatorname{ad}_{x}\right)=0$ for any $x$ in the Lie algebra $\mathfrak{g}$ of $G$. In the case of an almost abelian Lie group we have the following fact, which will prove very useful in forthcoming sections:

Proposition 2.3. [7] Let $G=\mathbb{R} \ltimes_{\phi} \mathbb{R}^{2 n+1}$ be an almost abelian Lie group. Then $G$ admits a lattice if and only if there exists a $t_{0} \neq 0$ such that $\phi\left(t_{0}\right)$ can be conjugated to an integer matrix.

In this situation, a lattice is given by $\Gamma=t_{0} \mathbb{Z} \ltimes P^{-1} \mathbb{Z}^{2 n+1}$, where $P \phi\left(t_{0}\right) P^{-1}$ is an integer matrix.

\section{Lattices in almost abelian Lie groups with LCK structures}

In this section we characterize firstly all almost abelian Lie algebras which admit LCK structures. Secondly, we determine which of the associated simply connected almost abelian Lie groups admit lattices, proving that this only happens in dimension 4.

\subsection{LCK almost abelian Lie algebras}

Let $\mathfrak{g}$ be an almost abelian Lie algebra of dimension $2 n+2$, so that there exists an abelian ideal $\mathfrak{u}$ of dimension $2 n+1$. Assume that $\mathfrak{g}$ is equipped with a Hermitian structure $(J,\langle\cdot, \cdot\rangle)$, where $J$ is a complex structure. Consider $\mathfrak{u} \cap J \mathfrak{u}$, the maximal $J$-invariant subspace of $\mathfrak{u}$. Clearly, $\operatorname{dim}(\mathfrak{u} \cap J \mathfrak{u})=2 n$, and there exists $f_{2} \in \mathfrak{u}$, $f_{2} \in(\mathfrak{u} \cap J \mathfrak{u})^{\perp}$, and $\left|f_{2}\right|=1$. Define $f_{1}=-J f_{2} \in \mathfrak{u}^{\perp}$. Then we get the orthogonal decomposition $\mathfrak{g}=\operatorname{span}\left\{f_{1}, f_{2}\right\} \oplus(\mathfrak{u} \cap J \mathfrak{u})$, where $\mathfrak{u}=\mathbb{R} f_{2} \oplus(\mathfrak{u} \cap J \mathfrak{u})$.

We can write also $\mathfrak{g}=\mathbb{R} f_{1} \ltimes \mathfrak{u}$, where the adjoint action of $f_{1}$ on $\mathfrak{u}$ is given by

$$
\left[f_{1}, f_{2}\right]=\mu f_{2}+v_{0},
$$

for some $v_{0} \in \mathfrak{u} \cap J \mathfrak{u}$ and $\mu \in \mathbb{R}$, and for $x \in \mathfrak{u} \cap J \mathfrak{u}$ we have

$$
\left[f_{1}, x\right]=\eta(x) f_{2}+A x, \quad \eta \in(\mathfrak{u} \cap J \mathfrak{u})^{*}, A \in \operatorname{End}(\mathfrak{u} \cap J \mathfrak{u}) .
$$

Since $J$ is integrable, we have that $N_{J}\left(f_{1}, x\right)=0$ for all $x \in \mathfrak{u} \cap J \mathfrak{u}$, that is,

$$
J\left[f_{1}, x\right]=\left[J f_{1}, x\right]+\left[f_{1}, J x\right]+J\left[J f_{1}, J x\right],
$$

which implies

$$
-\eta(x) f_{1}+J A x=\eta(J x) f_{2}+A J x,
$$

hence we obtain $\eta=0$ and $J A=A J$. Therefore, $\mathfrak{u} \cap J \mathfrak{u}$ is a $J$-invariant abelian ideal of codimension 2 in $\mathfrak{g}$. Denoting $\mathfrak{a}:=\mathfrak{u} \cap J \mathfrak{u}$, we obtain the following result (cf. [24]). 
Lemma 3.1. Let $\mathfrak{g}$ be an almost abelian Lie algebra and $(J,\langle\cdot, \cdot\rangle)$ a Hermitian structure on $\mathfrak{g}$. Then there exist a J-invariant abelian ideal $\mathfrak{a}$ of codimension 2 , an orthonormal basis $\left\{f_{1}, f_{2}\right\}$ of $\mathfrak{a}^{\perp}, v_{0} \in \mathfrak{a}$ and $\mu \in \mathbb{R}$ such that $\left[f_{1}, f_{2}\right]=\mu f_{2}+v_{0}$, $\left.\operatorname{ad}_{f_{1}}\right|_{\mathfrak{a}}$ commutes with $\left.J\right|_{\mathfrak{a}}$ and $\left.\operatorname{ad}_{f_{2}}\right|_{\mathfrak{a}}=0$.

We will assume from now on that $(J,\langle\cdot, \cdot\rangle)$ is LCK. Therefore there exists a closed 1 -form $\theta \neq 0$ such that $d \omega=\theta \wedge \omega$. We will consider two cases, according to the dimension of $\mathfrak{g}$.

3.1.1. Dimension of $\mathfrak{g} \geq 6 \quad$ In this case, that is, $n \geq 2$, we have that $\operatorname{dim}(\mathfrak{u} \cap J \mathfrak{u}) \geq$ 4.

Let us prove that $\theta$ is a multiple of the closed 1 -form $f^{1}$, where $f^{1}$ is the metric dual of $f_{1}$. Indeed, for each $x \in \mathfrak{u} \cap J \mathfrak{u}$ we can find $0 \neq y \in \mathfrak{u} \cap J \mathfrak{u}$ such that $\langle x, y\rangle=0=\langle x, J y\rangle$. Since $\mathfrak{u}$ is abelian, we have that $d \omega(x, y, J y)=0$, while on the other hand we compute $\theta \wedge \omega(x, y, J y)=\theta(x) \omega(y, J y)=\theta(x)|y|^{2}$, and as a consequence we obtain $\theta(x)=0$ for any $x \in \mathfrak{u} \cap J \mathfrak{u}$.

Now, from $d \omega\left(f_{2}, x, J x\right)=\theta \wedge \omega\left(f_{2}, x, J x\right)$ for $x \in \mathfrak{u} \cap J \mathfrak{u}, x \neq 0$, we obtain that $\theta\left(f_{2}\right)=0$. Consequently, $\theta=a f^{1}$ for some $a \neq 0$.

From $d \omega\left(f_{1}, f_{2}, x\right)=\theta \wedge \omega\left(f_{1}, f_{2}, x\right)$ and (10) we obtain that $\theta(x)=$ $-\left\langle J v_{0}, x\right\rangle$ for any $x \in \mathfrak{u} \cap J \mathfrak{u}$. This implies that $J v_{0}=0$ and therefore

$$
v_{0}=0, \text { so that }\left[f_{1}, f_{2}\right]=\mu f_{2} .
$$

This implies that $\mathfrak{g}=\mathfrak{a}^{\perp} \ltimes \mathfrak{a}$, where we are using the notation of Lemma 3.1.

Let us compute $d \omega\left(f_{1}, x, J y\right)=\theta \wedge \omega\left(f_{1}, x, J y\right)$ for $x, y \in \mathfrak{u} \cap J \mathfrak{u}$. We obtain that

$$
\langle A x, y\rangle+\langle x, A y\rangle=-a\langle x, y\rangle .
$$

Decomposing $A$ as $A=U+B$, where $U$ is self-adjoint and $B$ is skew-adjoint, it follows from the equation above that $U=-\frac{a}{2} \mathrm{Id}$, and therefore, setting $\lambda=-\frac{a}{2}$, we have that

$$
A=\lambda \mathrm{Id}+B, \quad B^{*}=-B, \quad B J=J B .
$$

Choosing an orthonormal basis $\left\{u_{1}, \ldots, u_{n}, v_{1}, \ldots, v_{n}\right\}$ of $\mathfrak{u} \cap J \mathfrak{u}$ such that $J u_{i}=v_{i}, i=1, \ldots, n$, we can identify $\mathfrak{u} \cap J \mathfrak{u}$ with $\mathbb{R}^{2 n}, \mathfrak{u}$ with $\mathbb{R}^{2 n+1}, \mathfrak{g}$ with $\mathbb{R} \ltimes \mathbb{R}^{2 n+1}$, and we have the following matrix representations:

$$
\left.J\right|_{\mathbb{R}^{2 n}}=\left(\begin{array}{cc}
0 & -I \\
I & 0
\end{array}\right),\left.\quad \operatorname{ad}_{f_{1}}\right|_{\mathbb{R}^{2 n+1}}=\left(\begin{array}{c}
\mu \mid \\
\\
\\
\mid \lambda I+B
\end{array}\right), \quad B \in \mathfrak{u}(n) .
$$

Moreover, the fundamental 2-form $\omega$ and the Lee form $\theta$ are given by:

$$
\omega=f^{1} \wedge f^{2}+\sum_{i=1}^{n} u^{i} \wedge v^{i}, \quad \theta=-2 \lambda f^{1},
$$


where $\left\{f^{1}, f^{2}, u^{1}, \ldots, u^{n}, v^{1}, \ldots, v^{n}\right\}$ is the dual basis. Note that the operator $\left.\operatorname{ad}_{f_{1}}\right|_{\mathbb{R}^{2 n+1}}$ is nilpotent if and only if it is zero, and that if $\mathfrak{g}$ is unimodular then $\lambda=-\frac{1}{2 n} \mu$.

Remarks 3.2. (i) When $\lambda=0$, it follows that $\theta=0$ and therefore the Hermitian structure $(J,\langle\cdot, \cdot\rangle)$ is Kähler. Since we are interested in non-Kähler LCK structures, we will assume $\lambda \neq 0$, and in this case, it is easy to see that the operator $\lambda \operatorname{Id}+B$ with $B \in \mathfrak{u}(n)$ is non-singular.

(ii) Almost Kähler structures on almost abelian Lie algebras were studied in [25].

3.1.2. Dimension of $\mathfrak{g}=4$ In this case we have $\mathfrak{g}=\operatorname{span}\left\{f_{1}, f_{2}\right\} \oplus \operatorname{span}\{u, v\}$, where $J f_{1}=f_{2}, J u=v$ and $\left\{f_{1}, f_{2}, u, v\right\}$ is an orthonormal basis of $\mathfrak{g}$. The brackets on $\mathfrak{g}$ are given by

$$
\begin{aligned}
& {\left[f_{1}, f_{2}\right]=\mu f_{2}+m u+n v, \text { for some } \mu, m, n \in \mathbb{R},} \\
& {\left[f_{1}, z\right]=A z, \text { where } z \in \operatorname{span}\{u, v\} \text { and } A=\left(\begin{array}{cc}
x & -y \\
y & x
\end{array}\right), \quad \text { with } x, y \in \mathbb{R} .}
\end{aligned}
$$

Then we get that

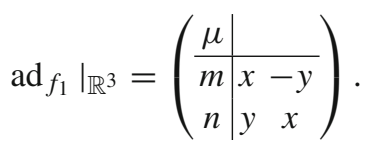

From $d \omega\left(f_{1}, f_{2}, u\right)=\theta \wedge \omega\left(f_{1}, f_{2}, u\right)$, we obtain that $\theta(u)=n$; in the same way, $\theta(v)=-m$. On the other hand, from $d \omega\left(f_{2}, z, J z\right)=\theta \wedge \omega\left(f_{2}, z, J z\right)$ for $z \in \operatorname{span}\{u, v\}$, we get that $\theta\left(f_{2}\right)=0$. Therefore we can write $\theta$ as

$$
\theta=a f^{1}+n u^{*}-m v^{*},
$$

for some $a \in \mathbb{R}$, where $\left\{f^{1}, f^{2}, u^{*}, v^{*}\right\}$ is the dual basis of $\left\{f_{1}, f_{2}, u, v\right\}$. Recalling that $\omega=f^{1} \wedge f^{2}+u^{*} \wedge v^{*}$, it follows from $d \omega=\theta \wedge \omega$ that $a=-2 x$.

Next, since $d \theta=0$, we obtain

$$
0=d \theta=-2 x d f^{1}+n d u^{*}-m d v^{*}=(-n x+m y) f^{1} \wedge u^{*}+(n y+m x) f^{1} \wedge v^{*},
$$

and we consider two cases according whether to $m^{2}+n^{2} \neq 0$ or $m^{2}+n^{2}=0$.

In the first case we have $x=y=0$, thus the only non-vanishing bracket is $\left[f_{1}, f_{2}\right]=\mu f_{2}+m u+n v$. If $\mu=0$, this Lie algebra is isomorphic to $\mathfrak{h}_{3} \times \mathbb{R}$, where $\mathfrak{h}_{3}$ is the 3-dimensional Heisenberg Lie algebra. It is well known that $\mathfrak{h}_{3} \times \mathbb{R}$ admits LCK structures ([10], see also [3,39]). If $\mu \neq 0$, this Lie algebra is isomorphic to $\mathfrak{a} \mathfrak{f} f(\mathbb{R}) \times \mathbb{R}^{2}$, where $\mathfrak{a f f}(\mathbb{R})$ denotes the non-abelian 2-dimensional Lie algebra of the group of affine motions of the real line. We point out that this decomposition is neither orthogonal nor $J$-invariant. Note that these Lie algebras have 1-dimensional commutator ideal, $\mathfrak{h}_{3} \times \mathbb{R}$ is nilpotent and $\mathfrak{a f f}(\mathbb{R}) \times \mathbb{R}^{2}$ is not unimodular.

The other case is $m=n=0$, so that

$$
\left.\operatorname{ad}_{f_{1}}\right|_{\mathbb{R}^{3}}=\left(\begin{array}{c|c}
\mu \mid & \\
\hline & \begin{array}{cc}
x & -y \\
y & x
\end{array}
\end{array}\right),
$$


and we obtain from (12) that $\theta=-2 x f^{1}$. Note that $\left.\operatorname{ad}_{f_{1}}\right|_{\mathbb{R}^{2}}=x I+B$, for some $x \in \mathbb{R}$ and $B \in \mathfrak{u}(1)$.

Conversely, it is easy to verify that if $\mathfrak{g}$ is an almost abelian Lie algebra where the adjoint action of $\mathbb{R}$ on the codimension one abelian ideal is given by either (11) or (13), then $\mathfrak{g}$ admits an LCK structure.

To close this section, we state the following result which summarizes the results obtained so far:

Theorem 3.3. Let $\mathfrak{g}$ be a $(2 n+2)$-dimensional almost abelian Lie algebra and $(J,\langle\cdot, \cdot\rangle)$ a Hermitian structure on $\mathfrak{g}$, and let $\mathfrak{g}^{\prime}$ denote the commutator ideal $[\mathfrak{g}, \mathfrak{g}]$ of $\mathfrak{g}$.

(i) If $\operatorname{dim} \mathfrak{g}^{\prime}=1$, then $(J,\langle\cdot, \cdot\rangle)$ is LCK if and only if $\mathfrak{g}$ is isomorphic to $\mathfrak{h}_{3} \times \mathbb{R}$ or $\mathfrak{a f f}(\mathbb{R}) \times \mathbb{R}^{2}$ as above.

(ii) If $\operatorname{dim} \mathfrak{g}^{\prime} \geq 2$, then $(J,\langle\cdot, \cdot\rangle)$ is $L C K$ if and only if $\mathfrak{g}$ can be decomposed as $\mathfrak{g}=\mathfrak{a}^{\perp} \ltimes \mathfrak{a}$, a J-invariant orthogonal sum with a codimension 2 abelian ideal $\mathfrak{a}$, and there exists an orthonormal basis $\left\{f_{1}, f_{2}\right\}$ of $\mathfrak{a}^{\perp}$ such that

$$
\left[f_{1}, f_{2}\right]=\mu f_{2}, \quad f_{2}=J f_{1},\left.\quad \operatorname{ad}_{f_{2}}\right|_{\mathfrak{a}}=0 \text { and }\left.\operatorname{ad}_{f_{1}}\right|_{\mathfrak{a}}=\lambda I+B,
$$

for some $\mu, \lambda \in \mathbb{R}, \lambda \neq 0$, and $B \in \mathfrak{u}(n)$. The corresponding Lee form is given by $\theta=-2 \lambda f^{1}$. Furthermore, the Lie algebra $\mathfrak{g}$ is unimodular if and only if $\lambda=-\frac{\mu}{2 n}$.

Remarks 3.4. (i) If we allow $\lambda=0$ in Theorem 3.3(ii), the Hermitian structures thus obtained are Kähler.

(ii) A Hermitian manifold is called Vaisman if it is LCK with parallel Lee form $\theta$. The left invariant LCK structures obtained on the Lie groups corresponding to the Lie algebras in Theorem 3.3(ii) or $\mathfrak{a f f}(\mathbb{R}) \times \mathbb{R}^{2}$ from Theorem 3.3(i) are never Vaisman. This can be seen either by a direct computation or by the fact that the endomorphisms $\operatorname{ad}_{f_{1}}$ are not skew-symmetric (see [3]). On the other hand, any LCK structure on $\mathfrak{h}_{3} \times \mathbb{R}$ from Theorem 3.3(i) is Vaisman (see [3,39]).

(iii) With the notation used in [2], the 4-dimensional Lie algebras admitting LCK structures obtained from Theorem 3.3(ii) correspond to $\mathfrak{r}_{3,1} \times \mathbb{R}, \mathfrak{r}_{3, \lambda}^{\prime} \times \mathbb{R}, \mathfrak{r}_{4, \mu, \mu}$ and $\mathfrak{r}_{4, \mu, \lambda}^{\prime}$ for some $\mu \neq 0$ and $\lambda \neq 0$. The Lie algebra $\mathfrak{a f f}(\mathbb{R}) \times \mathbb{R}^{2}$ from Theorem 3.3(i) is denoted by $\mathfrak{r}_{3,0} \times \mathbb{R}$ in [2].

\subsection{Lattices in the associated LCK Lie groups}

In this section, we will consider solvmanifolds associated to the almost abelian Lie algebras obtained in the previous section. Therefore we will study the existence of lattices in the simply connected Lie groups associated to these Lie algebras.

Recall that for an almost abelian solvable Lie group $G=\mathbb{R} \ltimes_{\phi} \mathbb{R}^{2 n+1}(n \geq 1)$, its Lie algebra is $\mathfrak{g}=\mathbb{R} \ltimes_{\mathrm{ad}_{f_{1}}} \mathbb{R}^{2 n+1}$ where $\mathbb{R}$ is generated by $f_{1}$, and the action is given by $\phi(t)=e^{t \operatorname{ad}_{f_{1}}}$.

Let $\mathfrak{g}$ be a unimodular almost abelian Lie algebra equipped with an LCK structure. If $\mathfrak{g}$ is nilpotent, then it follows from Theorem 3.3 that $\mathfrak{g}$ is isomorphic to $\mathfrak{h}_{3} \times \mathbb{R}$. 
The lattices in the associated simply connected nilpotent Lie group $H_{3} \times \mathbb{R}$ are well known (see [13] for their classification). The corresponding compact nilmanifolds are primary Kodaira surfaces [18].

From now on, we will consider non-nilpotent unimodular almost abelian Lie algebras with LCK structures. According to Theorem 3.3, such a Lie algebra $\mathfrak{g}$ can be decomposed as $\mathfrak{g}=\mathbb{R} f_{1} \ltimes \mathbb{R}^{2 n+1}$, an orthogonal sum, where $\mathbb{R}^{2 n+1}=\mathbb{R} f_{2} \oplus \mathbb{R}^{2 n}$ and

$$
\operatorname{ad}_{f_{1} \mid \mathbb{R}^{2 n+1}}=\left(\begin{array}{l|l}
\mu & \\
& -\frac{\mu}{2 n} I+B
\end{array}\right),
$$

for some $0 \neq \mu \in \mathbb{R}$ and $B \in \mathfrak{u}(n)$. Note that since $B$ is skew-symmetric, if $c \in \mathbb{C}$ is an eigenvalue of $A=-\frac{\mu}{2 n} I+B$, then $c=-\frac{\mu}{2 n} \pm i \eta$ for some $\eta \in \mathbb{R}$.

If $G$ denotes the simply connected almost abelian Lie group with Lie algebra $\mathfrak{g}$, then $G=\mathbb{R} \ltimes_{\phi} \mathbb{R}^{2 n+1}$ with

$$
\phi(t)=e^{\left.t \operatorname{ad}_{f_{1}}\right|_{\mathbb{R}^{2 n+1}}}=\left(\begin{array}{l}
e^{t \mu} \mid \\
\mid e^{-\frac{t \mu}{2 n}} e^{t B}
\end{array}\right) .
$$

This matrix has a real eigenvalue $e^{t \mu}$ and the others are $e^{-\frac{t \mu}{2 n} \pm i \eta}$ for some $\eta \in \mathbb{R}$.

The existence of lattices on $G$ will depend on the dimension of $G$, since we will show that such a lattice exists only if $\operatorname{dim} G=4$.

3.2.1. Lattices in dimension $\geq 6$ We will show that these Lie groups cannot admit lattices for $n \geq 2$. We state first a result about the roots of a certain class of polynomials with integer coefficients.

Lemma 3.5. Let $p$ be a polynomial of the form

$$
p(x)=x^{2 n+1}-m_{2 n} x^{2 n}+m_{2 n-1} x^{2 n-1}+\cdots+m_{1} x-1
$$

with $m_{j} \in \mathbb{Z}$ and $n \geq 2$, and let $x_{0}, \ldots, x_{2 n} \in \mathbb{C}$ denote the roots of $p$. If $x_{0} \in \mathbb{R}$ is a simple root and $\left|x_{1}\right|=\cdots=\left|x_{2 n}\right|$, then $x_{0}=1$ and $\left|x_{j}\right|=1, j=1, \ldots, 2 n$.

Proof. Let $\rho \in \mathbb{R}, \rho>0$, such that $\left|x_{j}\right|=\rho^{-1}$ for $j=1, \ldots, 2 n$. It follows from $\prod_{j=0}^{2 n} x_{j}=1$ that $\left|x_{0}\right|=\rho^{2 n}$.

Note that we may assume $\rho \geq 1$, since otherwise we consider the reciprocal polynomial $p^{*}(x):=-x^{2 n+1} p\left(x^{-1}\right)$.

Let us suppose that $\rho>1$.

We will prove first that $p$ is irreducible over $\mathbb{Z}[x]$. Indeed, if $p=q r$ with $q, r \in \mathbb{Z}[x]$, then $x_{0}$ will be a (simple) real root of one of these polynomials, say $q$, and therefore all the roots of $r$ have modulus $\rho^{-1}<1$. The coefficient $r(0)$ will be the product of these roots, hence $|r(0)|<1$, and this is a contradiction since $r(0) \in \mathbb{Z}-\{0\}$. 
Now, let us write $p(x)=\prod_{j=0}^{2 n}\left(x-x_{j}\right)$. Expanding this product we obtain:

$$
m_{2 n}=x_{0}+\sum_{j=1}^{2 n} x_{j}, \quad m_{1}=\frac{1}{x_{0}}+\sum_{j=1}^{2 n} \frac{1}{x_{j}} .
$$

Since $\sum_{j=1}^{2 n} \frac{1}{x_{j}}=\rho^{2} \sum_{j=1}^{2 n} x_{j}$, we obtain that

$$
m_{2 n}-x_{0}=\frac{1}{\rho^{2}}\left(m_{1}-\frac{1}{x_{0}}\right),
$$

which implies, recalling that $\left|x_{0}\right|=\rho^{2 n}$,

$$
\rho^{4 n+2}-m_{2 n} x_{0} \rho^{2}+m_{1} x_{0}-1=0 .
$$

We consider two different cases, according to: (i) $x_{0}=\rho^{2 n}$, or (ii) $x_{0}=-\rho^{2 n}$. (i) If $x_{0}=\rho^{2 n}$ then (15) becomes

$$
\rho^{4 n+2}-m_{2 n} \rho^{2 n+2}+m_{1} \rho^{2 n}-1=0 .
$$

Thus, $y_{0}:=\rho^{2}$ is a root of $q(x)=x^{2 n+1}-m_{2 n} x^{n+1}+m_{1} x^{n}-1$. Let $y_{1}, \ldots, y_{2 n}$ denote the other roots of $q$ and let us consider the polynomial $\tilde{q}(x)=\prod_{j=0}^{2 n}\left(x-y_{j}^{n}\right)$. Since $q(x)=\prod_{j=0}^{2 n}\left(x-y_{j}\right) \in \mathbb{Z}[x]$, it can be seen that $\tilde{q} \in \mathbb{Z}[x]$ as well.

Note that $\tilde{q}\left(\rho^{2 n}\right)=0$. Since $p, \tilde{q} \in \mathbb{Z}[x]$ are monic polynomials of the same degree, both vanish in $x_{0}=\rho^{2 n}$ and $p$ is irreducible, then $p=\tilde{q}$.

Therefore the set $\left\{y_{1}^{n}, \ldots, y_{2 n}^{n}\right\}$ is a permutation of $\left\{x_{1}, \ldots, x_{2 n}\right\}$, so that the polynomial $q$ has a simple real root $y_{0}=\rho^{2}>0$ and the other roots satisfy $\left|y_{1}\right|=\cdots=\left|y_{2 n}\right|=\rho^{-\frac{1}{n}}$. We can perform the same computations as above with the polynomial $q$, taking into account that in this case $\sum_{j=0}^{2 n} y_{j}=0$ and $\sum_{j=0}^{2 n} \frac{1}{y_{j}}=0$, since the coefficients of $x^{2 n}$ and $x^{1}$ in $q$ are 0 (here we are using that $n \geq 2$ ).

It follows that $\rho$ satisfies the equation

$$
\left(\rho^{\frac{1}{n}}\right)^{4 n+2}-1=0,
$$

so that $\rho=1$, which contradicts the assumption $\rho>1$.

(ii) If $x_{0}=-\rho^{2 n}$, then (15) becomes

$$
\rho^{4 n+2}+m_{2 n} \rho^{2 n+2}-m_{1} \rho^{2 n}-1=0 .
$$

Thus, $y_{0}:=\rho^{2}$ is a root of $q(x)=x^{2 n+1}+m_{2 n} x^{n+1}-m_{1} x^{n}-1$. Let $y_{1}, \ldots, y_{2 n}$ denote the other roots of $q$ and let us consider the polynomial $\tilde{q}(x)=\prod_{j=0}^{2 n}\left(x-y_{j}^{n}\right)$. Since $q(x)=\prod_{j=0}^{2 n}\left(x-y_{j}\right) \in \mathbb{Z}[x]$, then $\tilde{q} \in \mathbb{Z}[x]$ as well. Let us consider now the polynomial $q_{1} \in \mathbb{Z}[x]$ defined by $q_{1}(x)=-\tilde{q}(-x)$. Note that $q_{1}$ is a monic polynomial which vanishes in $x_{0}=-\rho^{2 n}$. It follows from the irreducibility of $p$ that $p=q_{1}$, but $p(0)=-1$ whereas $q_{1}(0)=1$, a contradiction. 
We conclude that the assumption $\rho>1$ leads to a contradiction, and as a consequence, we have $\rho=1$. Thus, $\left|x_{j}\right|=1$ for all $j=0, \ldots, 2 n$ and $x_{0}=1$ or $x_{0}=-1$.

If $x_{0}=-1$, then there exists $l \in\{0,1, \ldots, 2 n\}$ such that $x_{1}=\cdots=x_{2 l}=1$ and the remaining roots are non-real complex numbers $\alpha_{1}, \ldots, \alpha_{n-l}$ and their complex conjugates, with $\left|\alpha_{j}\right|=1$ for all $j$. However,

$$
1=\prod_{j=0}^{2 n} x_{j}=(-1)\left(\prod_{j=1}^{2 l} x_{j}\right)\left(\prod_{j=1}^{n-l}\left|\alpha_{j}\right|^{2}\right)=-1,
$$

a contradiction.

Therefore $x_{0}=1$ and $\left|x_{j}\right|=1$ for all $j$, and the theorem is proved.

Remark 3.6. It follows from Kronecker's theorem [23] that all the roots of the polynomial $p$ in Lemma 3.5 are roots of unity.

Theorem 3.7. If $G$ is as above with $\mu \neq 0$ and $\operatorname{dim} G \geq 6$, i.e. $n \geq 2$, then $G$ admits no lattice.

Proof. Suppose that $G$ admits lattices, from Proposition 2.3 there exists $t \neq 0$ such that $e^{t \operatorname{ad}_{f_{1}}}$ is conjugate to an integer matrix. Hence its characteristic polynomial $p$ has integer coefficients and it can be written as

$$
p(x)=x^{2 n+1}-m_{2 n} x^{2 n}+m_{2 n-1} x^{2 n-1}+\cdots+m_{1} x-1
$$

with $m_{j} \in \mathbb{Z}$. It follows from (14) that $p$ has a simple real root $x_{0}=e^{t \mu}$, and the other roots are complex with modulus $e^{-\frac{t \mu}{2 n}}$. It follows from Lemma 3.5 that $e^{t \mu}=1$. Since $\mu \neq 0$, then $t=0$, which is a contradiction.

3.2.2. Lattices in dimension 4 From Theorem 3.3 we have that $\mathfrak{g}=\mathbb{R} \ltimes \mathbb{R}^{3}$ and

$$
\operatorname{ad}_{f_{1} \mid \mathbb{R}^{3}}=\left(\begin{array}{c|cc}
\mu & & \\
\hline & -\frac{\mu}{2} & -y \\
y & -\frac{\mu}{2}
\end{array}\right) .
$$

We denote $\mathfrak{g}_{(\mu, y)}=(\mathfrak{g}, J,\langle\cdot, \cdot\rangle)$ where $\operatorname{ad}_{f_{1}}$ is given by (16). In the non-Kähler case, i.e. $\mu \neq 0$, we get that $\mathfrak{g}_{(\mu, y)}$ is isomorphic to $\mathfrak{g}_{\left(1, \frac{y}{\mu}\right)}$. We denote this Lie algebra by $\mathfrak{g}_{b}$, where $b=\frac{y}{\mu}$.

Remark 3.8. Note that $\mathfrak{g}_{b}$ and $\mathfrak{g}_{-b}$ are isomorphic. Moreover, for $b \neq 0, \mathfrak{g}_{b}$ is isomorphic to $\mathfrak{r}_{4,1 / b,-1 / 2 b}^{\prime}$ and $\mathfrak{g}_{0}$ is isomorphic to $\mathfrak{r}_{4,-\frac{1}{2},-\frac{1}{2}}$ from [2], and it follows that they are not pairwise isomorphic for $b \geq 0$.

Let us assume that the simply connected Lie group $G_{b}$ associated to $\mathfrak{g}_{b}$ admits lattices. Then according to Proposition 2.3 there exists $t_{0} \in \mathbb{R}, t_{0} \neq 0$, such that $e^{t_{0} \operatorname{ad}_{f_{1}}}$ is conjugated to a matrix with integer coefficients. Therefore the characteristic polynomial of $e^{t_{0} \text { ad }} f_{1}$ is

$$
f(x)=x^{3}-m x^{2}+n x-1,
$$


with $m, n \in \mathbb{Z}$. Note that $f$ has a simple real root $e^{t_{0}} \neq 1$ and two complex conjugate roots $e^{t_{0}\left(-\frac{1}{2} \pm i b\right)} \in \mathbb{C}-\mathbb{R}$. Indeed, if $e^{t_{0}\left(-\frac{1}{2} \pm i b\right)} \in \mathbb{R}$, then $e^{-\frac{t_{0}}{2}}$ (or its opposite) is a double real root, and it is easy to see that this implies $e^{-\frac{t_{0}}{2}}=1$ and therefore $t_{0}=0$, a contradiction. In particular, $G_{0}$ does not admit lattices.

Conversely, we consider $f(x)=x^{3}-m x^{2}+n x-1$ with $m, n \in \mathbb{Z}$ such that $f$ has a simple real root $c \neq 1$ and two complex conjugate roots $\alpha, \bar{\alpha} \in \mathbb{C}-\mathbb{R}$. Then $|\alpha|^{2} c=1$, so that $c>0$. If $\alpha=|\alpha| e^{i \phi}$ with $\phi \in(0, \pi)$ we consider the Lie algebra $\mathfrak{g}=\mathbb{R} \ltimes \mathbb{R}^{3}$ where the action is given by

$$
\left.\operatorname{ad}_{f_{1}}\right|_{\mathbb{R}^{3}}=\left(\begin{array}{c|cc}
-2 \log |\alpha| & & \\
\hline & \left.\begin{array}{cc}
\log |\alpha| & -\phi \\
\phi & \log |\alpha|
\end{array}\right) .
\end{array}\right.
$$

Then

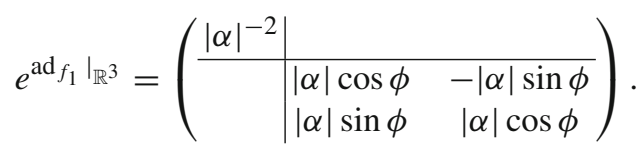

Since this matrix has eigenvalues $c, \alpha$ and $\bar{\alpha}$ and they are all different, we have that it is conjugated to the companion matrix

$$
\left(\begin{array}{ccc}
0 & 0 & 1 \\
1 & 0 & -n \\
0 & 1 & m
\end{array}\right) \in S L(3, \mathbb{Z})
$$

According to Proposition 2.3 the simply connected Lie group associated to $\mathfrak{g}$ admits lattices.

Note that as $c \neq 1$, this Lie algebra coincides with $\mathfrak{g}_{(-2 \log |\alpha|, \phi)}$ as above and therefore is isomorphic to $\mathfrak{g}_{b}$ with $b=\frac{\phi}{\log c}$.

Let $\Sigma=\left\{(m, n) \in \mathbb{Z} \times \mathbb{Z}: f_{m, n}(x)=x^{3}-m x^{2}+n x-1\right.$ has roots $c \in$ $\mathbb{R}, \alpha, \bar{\alpha} \in \mathbb{C}-\mathbb{R}\}$. This region $\Sigma$ is the set of the pairs $(m, n) \in \mathbb{Z} \times \mathbb{Z}$ such that the discriminant $\Delta_{m, n}$ of $f_{m, n}$ is negative, that is, $\Delta_{m, n}=-27-4 m^{3}+18 m n+$ $m^{2} n^{2}-4 n^{3}<0$ (see Fig. 1).

Note that $c=1$ if and only if $m=n$, and in this case the other roots are complex conjugate if and only if $m=0,1,2$.

Let $\Sigma^{\prime}=\Sigma-\{(0,0),(1,1),(2,2)\}$ and for any $k \in \mathbb{Z}$ consider the function $h_{k}: \Sigma^{\prime} \rightarrow \mathbb{R}$ that assigns to $(m, n)$ the real number $\frac{\phi_{k}}{\log c}$ where $\phi_{k}=\phi+2 k \pi$, $\phi \in(0, \pi)$. With this notation, we may state the following result.

Theorem 3.9. The simply connected almost abelian Lie group $G_{b}$ with Lie algebra $\mathfrak{g}_{b}$ admits lattices if and only if $b \in \bigcup_{k \in \mathbb{Z}} \operatorname{Im}\left(h_{k}\right)$, a countable subset of $\mathbb{R}$.

Remarks 3.10. (i) The solvmanifolds associated to $\mathfrak{g}_{b}$ are Inoue surfaces of type $S^{0}$ $[21,41]$ (see [40] for an explicit construction of a lattice in the Lie groups $G_{b}$ ).

(ii) Note that if $(m, n) \in \Sigma$ then $(n, m) \in \Sigma$ as well, since $f_{n, m}(x)=-x^{3} f_{m, n}\left(\frac{1}{x}\right)$. Thus $\Sigma$ is symmetric with respect to the diagonal $y=x$. 


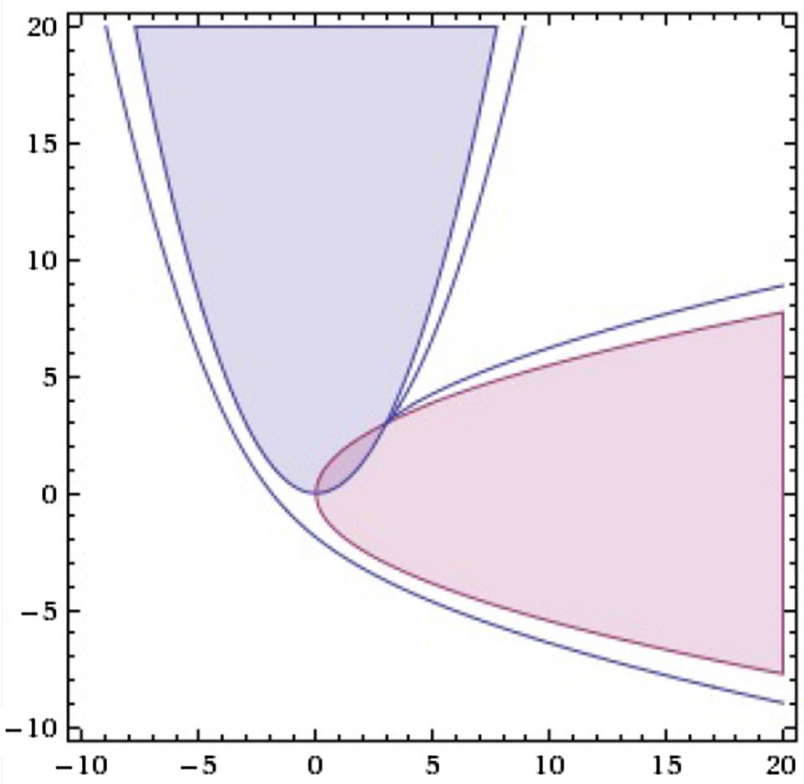

Fig. 1. Discriminant of $f_{m, n}<0$

(iii) The region $\Sigma$ contains all pairs $(m, n)$ such that $m^{2}<3 n$ or $n^{2}<3 m$ (see Fig. 1).

(iv) It is easy to see that for $(m, n) \in \Sigma$, the real root $c$ of $f_{m, n}$ satisfies $c>1$ when $n<m$, therefore $c$ is a Pisot number. Recall that a Pisot number is a real algebraic number greater than 1 such that all others roots of its minimal polynomial have absolute value less than 1 . Similarly, if $n>m$, then $c^{-1}$ is a Pisot number. More precisely, these Pisot numbers belong to the class $\mathcal{P}$ considered in [8].

Example 3.11. For $m=0, n=3$, it can be seen that the possible values of $b$ are

$$
b=\left|\frac{\arctan \left(\frac{\sqrt{3}\left(r^{2}+1\right)}{r^{2}-1}\right)+2 k \pi}{\log \left(r-r^{-1}\right)}\right|, \quad r=\left(\frac{1+\sqrt{5}}{2}\right)^{\frac{1}{3}}, \quad k \in \mathbb{Z} .
$$

\section{Lattices in almost abelian Lie groups with LCS structures}

In this section we begin by characterizing locally conformal symplectic structures on almost abelian Lie algebras. Then we study the existence of lattices in the associated simply connected almost abelian Lie groups: in dimension 4 we determine all these Lie groups, and in each higher dimension we exhibit an almost abelian Lie group with a countable family of non isomorphic lattices. 


\subsection{LCS almost abelian Lie algebras}

Let $(\omega, \theta)$ denote an LCS structure on an almost abelian Lie algebra $\mathfrak{g}$ of dimension $2 n+2$. If $\mathfrak{u} \subset \mathfrak{g}$ is an abelian ideal of $\mathfrak{g}$ of codimension 1 , then $\left.\omega\right|_{\mathfrak{u} \times \mathfrak{u}}$ has rank $2 n$. Therefore there exist $f_{2} \in \mathfrak{u}$ and a vector subspace $\mathfrak{v} \subset \mathfrak{u}$ such that $\mathfrak{u}=\mathbb{R} f_{2} \oplus \mathfrak{v}$, $\omega\left(f_{2}, \mathfrak{v}\right)=0$ and $\left.\omega\right|_{\mathfrak{v} \times \mathfrak{v}}$ is non degenerate. Since $\omega$ is non degenerate on $\mathfrak{g}$, there exists $f_{1} \in \mathfrak{g}$ such that $\omega\left(f_{1}, f_{2}\right)=1$ and

$$
\mathfrak{g}=\mathbb{R} f_{1} \oplus \mathfrak{u}
$$

The adjoint action of $f_{1}$ on $\mathfrak{u}$ is given by

$$
\left[f_{1}, f_{2}\right]=\mu f_{2}+v_{0}
$$

for some $v_{0} \in \mathfrak{v}$ and $\mu \in \mathbb{R}$, and for $x \in \mathfrak{v}$ we have

$$
\left[f_{1}, x\right]=\eta(x) f_{2}+A x, \quad \text { with } \eta \in \mathfrak{v}^{*}, A \in \operatorname{End}(\mathfrak{v}) .
$$

Since $\omega$ is non degenerate on $\mathfrak{v}$, there exists a basis $\left\{u_{1}, \ldots, u_{n}, v_{1}, \ldots, v_{n}\right\}$ of $\mathfrak{v}$ such that

$$
\omega=f^{1} \wedge f^{2}+\sum_{i=1}^{n} u^{i} \wedge v^{i}
$$

where $\left\{f^{1}, f^{2}, u^{1}, \ldots, u^{n}, v^{1}, \ldots, v^{n}\right\}$ is the dual basis of $\mathfrak{g}^{*}$. We can identify $\mathfrak{v}$ with $\mathbb{R}^{2 n}, \mathfrak{u}$ with $\mathbb{R}^{2 n+1}, \mathfrak{g}$ with $\mathbb{R} \ltimes \mathbb{R}^{2 n+1}$, and if we denote $M=\left.\operatorname{ad}_{f_{1}}\right|_{\mathbb{R}^{2 n+1}}$, then we can express $M$ as

$$
M=\left(\begin{array}{c|c}
\mu & w^{t} \\
\hline v_{0} & A
\end{array}\right),
$$

where $w^{t}=\left(\eta\left(u_{1}\right), \ldots, \eta\left(u_{n}\right), \eta\left(v_{1}\right), \ldots, \eta\left(v_{n}\right)\right)$.

We will consider two different cases, depending on whether the dimension of $\mathfrak{g}$ is 4 or greater than 4 . Unlike the locally conformal Kähler case, the description of the Lie algebras admitting LCS forms in dimension 4 will be different from those Lie algebras in higher dimensions.

4.1.1. Dimension of $\mathfrak{g} \geq 6$ In this case, we have that $\operatorname{dim} \mathfrak{v} \geq 4$. We will see next that the corresponding Lee form $\theta$ is given by $\theta=a f^{1}$ for some $a \in \mathbb{R}, a \neq 0$, and the vector $v_{0}$ in (18) is $v_{0}=0$.

Since $\omega$ is non degenerate on $\mathfrak{v}$, for any nonzero $x \in \mathfrak{v}$, there exist $x^{\prime}, y, y^{\prime} \in \mathfrak{v}$ such that $\omega(x, y)=\omega\left(x, y^{\prime}\right)=0$ and $\omega\left(x, x^{\prime}\right)=\omega\left(y, y^{\prime}\right)=1$. Then:

$\diamond d \omega\left(x, y, y^{\prime}\right)=\theta \wedge \omega\left(x, y, y^{\prime}\right)$ implies that $\theta(x)=0$, so that $\left.\theta\right|_{\mathfrak{v}}=0$;

$\diamond d \omega\left(f_{2}, x, x^{\prime}\right)=\theta \wedge \omega\left(f_{2}, x, x^{\prime}\right)$ and the fact that $\left.\theta\right|_{\mathfrak{v}}=0$ imply that $\theta\left(f_{2}\right)=0$. 
Thus $\theta=a f^{1}$ for some $a \in \mathbb{R}, a \neq 0$.

We compute next $d \omega\left(f_{1}, f_{2}, x\right)=\theta \wedge \omega\left(f_{1}, f_{2}, x\right)$. We have that

$$
\begin{aligned}
d \omega\left(f_{1}, f_{2}, x\right) & =-\omega\left(\left[f_{1}, f_{2}\right], x\right)-\omega\left(\left[x, f_{1}\right], f_{2}\right) \\
& =-\omega\left(\mu f_{2}+v_{0}, x\right)+\omega\left(\eta(x) f_{2}+A x, f_{2}\right) \\
& =-\omega\left(v_{0}, x\right)
\end{aligned}
$$

since $\omega\left(f_{2}, \mathfrak{v}\right)=0$ and $\mathfrak{u}$ is abelian. On the other hand, $\theta \wedge \omega\left(f_{1}, f_{2}, x\right)=$ $\theta\left(f_{1}\right) \omega\left(f_{2}, x\right)=0$ since $\theta=a f^{1}$. As a consequence, $\omega\left(v_{0}, x\right)=0$ for all $x \in \mathfrak{v}$. Since $\omega$ is non degenerate on $\mathfrak{v}$, it follows that $v_{0}=0$.

Next we take into account $d \omega\left(f_{1}, x, y\right)=\theta \wedge \omega\left(f_{1}, x, y\right)$ for $x, y \in \mathfrak{v}$. Since $\theta=a f^{1}$, the right-hand side is $\omega\left(f_{1}, x, y\right)=a \omega(x, y)$. On the other hand,

$$
\begin{aligned}
d \omega\left(f_{1}, x, y\right) & =-\omega\left(\left[f_{1}, x\right], y\right)-\omega\left(\left[y, f_{1}\right], x\right) \\
& =-\omega\left(\eta(x) f_{2}+A x, y\right)-\omega\left(x, \eta(y) f_{2}+A y\right) \\
& =-\omega(A x, y)-\omega(x, A y),
\end{aligned}
$$

since $f_{2}$ is $\omega$-orthogonal to $\mathfrak{v}$. Let us decompose $A=U+B$, with $U^{*_{\omega}}=U$ and $B^{*_{\omega}}=-B$. Recall that given a linear transformation $T$ on the symplectic vector space $\left(\mathfrak{v},\left.\omega\right|_{\mathfrak{v} \times \mathfrak{v}}\right)$, its $\omega$-adjoint $T^{*_{\omega}}$ is defined by $\omega(T u, v)=\omega\left(u, T^{* \omega} v\right)$ for any $u, v \in \mathfrak{v}$. Note that $B^{* \omega}=-B$ means that $B \in \mathfrak{s p}\left(\mathfrak{v},\left.\omega\right|_{\mathfrak{v} \times \mathfrak{v}}\right) \simeq \mathfrak{s p}(n, \mathbb{R})$.

With this decomposition the equation above becomes $d \omega\left(f_{1}, x, y\right)=-2 \omega$ $(U x, y)$, hence

$$
-2 \omega(U x, y)=a \omega(x, y) \text { for all } x, y \in \mathfrak{v} \text {. }
$$

It follows that $U=-\frac{a}{2}$ Id and

$$
A=-\frac{a}{2} \mathrm{Id}+B, \quad \text { with } B \in \mathfrak{s p}(n, \mathbb{R}) .
$$

Therefore $M$ has the following matrix representation with respect to the basis above:

$$
M=\left(\begin{array}{c|c}
\mu & w^{t} \\
\hline 0 & -\frac{a}{2} I+B
\end{array}\right),
$$

with $B \in \mathfrak{s p}(n, \mathbb{R})$. Summarizing, we have the following result:

Theorem 4.1. Let $\mathfrak{g}$ be an $(2 n+2)$-dimensional almost abelian Lie algebra with $\operatorname{dim} \mathfrak{g} \geq 6$. Then $\mathfrak{g}$ admits an LCS structure if and only if $\mathfrak{g}$ is isomorphic to $\mathbb{R} \ltimes_{M} \mathbb{R}^{2 n+1}$, where the adjoint action of $\mathbb{R}$ on $\mathbb{R}^{2 n+1}$ is given by

$$
M=\left(\begin{array}{c|c}
\mu & w^{t} \\
\hline 0 & \lambda I+B
\end{array}\right),
$$


for some $\mu, \lambda \in \mathbb{R}, \lambda \neq 0, w \in \mathbb{R}^{2 n}$ and $B \in \mathfrak{s p}(n, \mathbb{R})$. The LCS form is $\omega=$ $f^{1} \wedge f^{2}+\sum_{i=1}^{n} u^{i} \wedge v^{i}$, and the Lee form is $\theta=-2 \lambda f^{1}$. Moreover, $\mathfrak{g}$ is unimodular if and only if $\lambda=-\frac{\mu}{2 n}$.

Remarks 4.2. (i) In the same way, it can be seen that any symplectic almost abelian Lie algebra is isomorphic to $\mathbb{R} \ltimes_{M} \mathbb{R}^{2 n+1}$ with $M$ as in (20) with $\lambda=0$ (cf. [25]). (ii) When $w=0$ and $B \in \mathfrak{u}(n) \subset \mathfrak{s p}(n, \mathbb{R})$, the LCS form is in fact LCK. Indeed, in the notation of the theorem, the almost complex structure $J$ defined by $J f_{1}=f_{2}, J u_{i}=v_{i}, i=1, \ldots, n$, is integrable and the metric $\langle\cdot, \cdot\rangle=\omega(\cdot, J \cdot)$ is compatible with $J$.

In the following result we show that the LCS structures constructed in Theorem 4.1 are all of the second kind. Therefore this theorem provides a way of producing many examples of Lie algebras with this type of structures.

Corollary 4.3. Let $\mathfrak{g}$ be an almost abelian Lie algebra with $\operatorname{dim} \mathfrak{g} \geq 6$. If $\mathfrak{g}$ admits an LCS structure, then it is of the second kind.

Proof. Let $(\omega, \theta)$ be the LCS structure on $\mathfrak{g}$. From Theorem 4.1 we have that $\theta=-2 \lambda f^{1}$. Recall that the LCS structure is of the second kind if $\left.\theta\right|_{\mathfrak{g}_{\omega}} \equiv 0$, where $\mathfrak{g}_{\omega}$ is given by (5).

Let $x \in \mathfrak{g}_{\omega}, x=c f_{1}+v$ with $c \in \mathbb{R}$ and $v \in \mathbb{R}^{2 n+1}$. We compute $d \omega(x, y, z)=$ $\theta \wedge \omega(x, y, z)$ for $y, z \in \mathbb{R}^{2 n+1}$. Since $x \in \mathfrak{g}_{\omega}$ we have $c \lambda \omega(y, z)=0$ for all $y, z \in \mathbb{R}^{2 n+1}$. Since $\omega$ is non degenerate and $\lambda \neq 0$ we obtain $c=0$. Therefore $\left.\theta\right|_{\mathfrak{g}_{\omega}}$ is identically zero, hence the LCS structure is of the second kind.

4.1.2. Dimension of $\mathfrak{g}=4$ In this section we will proceed in a different way to determine the 4-dimensional almost abelian Lie algebras admitting an LCS structure.

Let $\mathfrak{g}$ be a 4-dimensional almost abelian Lie algebra equipped with an LCS structure, $\mathfrak{g}=\mathbb{R} \ltimes_{M} \mathbb{R}^{3}$, where $M$ denotes the adjoint action of $\mathbb{R}$ on $\mathbb{R}^{3}$, and let $\mu$ be a real eigenvalue of $M$. Then we have

$$
M=\left(\begin{array}{c|c}
\mu & w^{t} \\
\hline 0 & A
\end{array}\right),
$$

where $A \in \mathfrak{g l}(2, \mathbb{R})$ for some basis $\left\{f_{1}, f_{2}, f_{3}, f_{4}\right\}$.

Lemma 4.4. With notation as above, if $\operatorname{tr}(A) \neq 0$ then $\mathfrak{g}$ admits an LCS structure.

Proof. It is easy to see that $\omega=f^{1} \wedge f^{2}+f^{3} \wedge f^{4}$ and $\theta=-\operatorname{tr}(A) f^{1}$ satisfy $d \omega=\theta \wedge \omega$ and $d \theta=0$, where $\left\{f^{1}, f^{2}, f^{3}, f^{4}\right\}$ is the dual basis of $\mathfrak{g}^{*}$. 
Given $\mathfrak{g}=\mathbb{R} \ltimes_{M} \mathbb{R}^{3}$, according to Lemma 2.1, we may assume that $M$ is in its canonical Jordan form, up to scaling. In this case, there are four different possibilities for $M$ :

$$
\begin{aligned}
M_{1} & =\left(\begin{array}{lll}
\lambda_{1} & & \\
& \lambda_{2} & \\
& & \lambda_{3}
\end{array}\right), M_{2}^{\mu, \lambda}=\left(\begin{array}{lll}
\mu & & \\
& \lambda & 1 \\
& & \lambda
\end{array}\right), M_{3}^{\mu}=\left(\begin{array}{ccc}
\mu & 1 & \\
& \mu & 1 \\
& & \mu
\end{array}\right), \\
M_{4}^{\mu, \lambda} & =\left(\begin{array}{ccc}
\mu & & \\
& \lambda & -1 \\
& 1 & \lambda
\end{array}\right),
\end{aligned}
$$

for $\lambda, \mu, \lambda_{i} \in \mathbb{R}, \lambda_{1}^{2}+\lambda_{2}^{2}+\lambda_{3}^{2} \neq 0$.

The only cases that are not covered by Lemma 4.4 are the following:

$$
M_{2}^{0,0}=\left(\begin{array}{ccc}
0 & & \\
& 0 & 1 \\
& & 0
\end{array}\right), \quad M_{3}^{0}=\left(\begin{array}{ccc}
0 & 1 & \\
& 0 & 1 \\
& & 0
\end{array}\right), \quad M_{4}^{\mu, 0}=\left(\begin{array}{ccc}
\mu & & \\
& 0 & -1 \\
& 1 & 0
\end{array}\right) .
$$

By a direct computation it can be seen that $\mathfrak{g}=\mathbb{R} \ltimes_{M} \mathbb{R}^{3}$ admits LCS structures for $M=M_{2}^{0,0}, M_{3}^{0}, M_{4}^{0,0}$, whereas for $M=M_{4}^{\mu, 0}$ with $\mu \neq 0$ it does not admit any. We can summarize these results in the next theorem, where we use the notation from [2].

Theorem 4.5. Let $\mathfrak{g}$ be a 4-dimensional almost abelian Lie algebra with an LCS structure. Then $\mathfrak{g}$ is isomorphic to one of the following Lie algebras:

$$
\begin{aligned}
\mathfrak{h}_{3} \times \mathbb{R}:\left[e_{1}, e_{2}\right] & =e_{3} \\
\mathfrak{n}_{4}:\left[e_{1}, e_{2}\right] & =e_{3},\left[e_{1}, e_{3}\right]=e_{4} \\
\mathfrak{r}_{3, \lambda} \times \mathbb{R}:\left[e_{1}, e_{2}\right] & =e_{2},\left[e_{1}, e_{3}\right]=\lambda e_{3} \\
\mathfrak{r}_{4, \mu, \lambda}:\left[e_{1}, e_{2}\right] & =e_{2},\left[e_{1}, e_{3}\right]=\mu e_{3},\left[e_{1}, e_{4}\right]=\lambda e_{4}, \mu \lambda \neq 0 \\
\mathfrak{r}_{3} \times \mathbb{R}:\left[e_{1}, e_{2}\right] & =e_{2},\left[e_{1}, e_{3}\right]=e_{2}+e_{3} \\
\mathfrak{r}_{4, \lambda}:\left[e_{1}, e_{2}\right] & =e_{2},\left[e_{1}, e_{3}\right]=\lambda e_{3},\left[e_{1}, e_{4}\right]=e_{3}+\lambda e_{4} \\
\mathfrak{r}_{4}:\left[e_{1}, e_{2}\right] & =e_{2},\left[e_{1}, e_{3}\right]=e_{2}+e_{3},\left[e_{1}, e_{4}\right]=e_{3}+e_{4} \\
\mathfrak{r}_{3, \lambda}^{\prime} \times \mathbb{R}:\left[e_{1}, e_{2}\right] & =\lambda e_{2}-e_{3},\left[e_{1}, e_{3}\right]=e_{2}+\lambda e_{3} \\
\mathfrak{r}_{4, \mu, \lambda}^{\prime}:\left[e_{1}, e_{2}\right] & =\mu e_{2},\left[e_{1}, e_{3}\right]=\lambda e_{3}-e_{4},\left[e_{1}, e_{4}\right]=e_{3}+\lambda e_{4}, \mu \neq 0, \lambda \neq 0
\end{aligned}
$$

Proof. We prove first that, as mentioned above, $\mathfrak{g}=\mathbb{R} f_{1} \ltimes_{M} \mathbb{R}^{3}$ does not admit LCS structures for $M=M_{4}^{\mu, 0}$ with $\mu \neq 0$. Let $\left\{f^{1}, f^{2}, f^{3}, f^{4}\right\}$ be the dual basis of $\mathfrak{g}^{*}$, and let us assume that $(\omega, \theta)$ is an LCS structure on $\mathfrak{g}$, where

$$
\begin{aligned}
& \omega=a_{1} f^{1} \wedge f^{2}+a_{2} f^{1} \wedge f^{3}+a_{3} f^{1} \wedge f^{4}+a_{4} f^{2} \wedge f^{3}+a_{5} f^{2} \wedge f^{4}+a_{6} f^{3} \wedge f^{4} \\
& \theta=b_{1} f^{1}+b_{2} f^{2}+b_{3} f^{3}+b_{4} f^{4}
\end{aligned}
$$

for some $a_{i}, b_{j} \in \mathbb{R}$. Since $\theta$ is closed, we have that $b_{2}=b_{3}=b_{4}=0$. Computing $d \omega=\theta \wedge \omega$ and using that $\theta \neq 0$ we have $a_{6}=0, b_{1} a_{4}=-\mu a_{4}+a_{5}$ and 
$b_{1} a_{5}=-a_{4}-\mu a_{5}$. Therefore $a_{4}=a_{5}=0$, which is a contradiction with the fact that $\omega$ is non degenerate.

To finish the proof we refer to Table 1, where LCS structures for all the Lie algebras in the statement are exhibited.

Remarks 4.6. (i) The Lie algebra $\mathfrak{g}=\mathbb{R} \ltimes_{M_{4}^{0,0}} \mathbb{R}^{3}$ corresponds to the Lie algebra $\mathfrak{r}_{3,0}^{\prime} \times \mathbb{R}$, while $\mathfrak{g}=\mathbb{R} \ltimes_{M_{4}^{\mu, 0}} \mathbb{R}^{3}, \mu \neq 0$, corresponds to $\mathfrak{r}_{4, \mu, 0}^{\prime}$.

(ii) Among the Lie algebras in Theorem 4.5, the unimodular ones are: $\mathfrak{h}_{3} \times \mathbb{R}, \mathfrak{n}_{4}$, $\mathfrak{r}_{3,-1} \times \mathbb{R}, \mathfrak{r}_{4, \lambda,-(1+\lambda)}, \mathfrak{r}_{4,-\frac{1}{2}}, \mathfrak{r}_{3,0}^{\prime} \times \mathbb{R}$ and $\mathfrak{r}_{4, \lambda,-\lambda / 2}^{\prime}$

(iii) According to [36], the Lie algebras $\mathfrak{r}_{3, \lambda} \times \mathbb{R}(\lambda \neq 0,-1), \mathfrak{r}_{4, \mu, \lambda}(\mu \neq-\lambda$ or $\mu \neq-1), \mathfrak{r}_{3} \times \mathbb{R}, \mathfrak{r}_{4, \lambda}(\lambda \neq 0,-1), \mathfrak{r}_{4}, \mathfrak{r}_{3, \lambda}^{\prime} \times \mathbb{R}(\lambda \neq 0)$ and $\mathfrak{r}_{4, \mu, \lambda}^{\prime}$ do not admit any symplectic structures.

(iv) Note that $\mathfrak{r}_{3,-1}$ is the Lie algebra $\mathfrak{e}(1,1)$ (it is also denoted by $\mathfrak{s o l}^{3}$ ) of the group of rigid motions of Minkowski 2-space, and $\mathfrak{r}_{3,0}^{\prime}$ is the Lie algebra $\mathfrak{e}(2)$ of the group of rigid motions of Euclidean 2-space.

Next, we will study whether the LCS structures on these 4-dimensional almost abelian Lie algebras are of first or second kind. We prove first a result which holds in any dimension.

Lemma 4.7. Let $\mathfrak{g}=\mathbb{R} f_{1} \ltimes_{M} \mathfrak{u}$ be an almost abelian Lie algebra as in (17) endowed with an LCS structure $(\omega, \theta)$. If $M$ is invertible then such LCS structure is of the second kind.

Proof. Indeed, if $M$ is invertible then $[\mathfrak{g}, \mathfrak{g}]=\mathfrak{u}$. Since $\theta([\mathfrak{g}, \mathfrak{g}])=0$, it follows that $\theta=c f^{1}$ for some $c \in \mathbb{R}, c \neq 0$. Let $z=a f_{1}+v \in \mathfrak{g}_{\omega}$ with $a \in \mathbb{R}, v \in \mathfrak{u}$. Computing $d \omega(z, x, y)=\theta \wedge \omega(z, x, y)$ for all $x, y \in \mathfrak{u}$, we obtain that $a=0$ and therefore $\mathfrak{g}_{\omega} \subset \mathfrak{u}$.

Let $\mathfrak{g}=\mathbb{R} \ltimes_{M} \mathbb{R}^{3}$ be a 4-dimensional almost abelian Lie algebra equipped with an LCS structure. The cases included in the lemma above correspond to: $\mathfrak{r}_{4, \mu, \lambda}, \mathfrak{r}_{4, \lambda}$ $(\lambda \neq 0), \mathfrak{r}_{4}$ and $\mathfrak{r}_{4, \mu, \lambda}^{\prime}, \lambda \neq 0$. Therefore any LCS structure on these Lie algebras is of the second kind.

Let us suppose now that $M$ is not invertible. It was proved in [6] that any LCS structure on a nilpotent Lie algebra is of the first kind, so that we may assume that $M$ is not nilpotent. The Jordan form of such a matrix can be one of the following:

$$
\begin{aligned}
M_{1} & =\left(\begin{array}{lll}
\lambda_{1} & & \\
& \lambda_{2} & \\
& & \lambda_{3}
\end{array}\right) \text { with } \lambda_{1} \lambda_{2} \lambda_{3}=0, \quad M_{2}^{0, \lambda}=\left(\begin{array}{lll}
0 & & \\
& \lambda & 1 \\
& \lambda
\end{array}\right) \text { with } \lambda \neq 0, \\
M_{2}^{\mu, 0} & =\left(\begin{array}{lll}
\mu & & \\
& 0 & 1 \\
& & 0
\end{array}\right) \text { with } \mu \neq 0, \quad M_{4}^{0, \lambda}=\left(\begin{array}{ccc}
0 & & \\
& \lambda & -1 \\
& 1 & \lambda
\end{array}\right) .
\end{aligned}
$$

By direct computations we can verify that all these Lie algebras admit both LCS structures of the first kind and of the second kind, except for the case $M_{4}^{0,0}$, which admits only LCS structures of the first kind. This Lie algebra corresponds to $\mathfrak{r}_{3,0}^{\prime} \times \mathbb{R}$ in Theorem 4.5 . 
Table 1. LCS structures of the first or second kind on 4-dimensional Lie algebras

\begin{tabular}{|c|c|c|}
\hline Lie algebra & LCS first kind & LCS second kind \\
\hline $\mathfrak{h}_{3} \times \mathbb{R}$ & $\begin{array}{l}\omega=e^{1} \wedge e^{2}+e^{3} \wedge e^{4} \\
\theta=-e^{4}\end{array}$ & \\
\hline $\mathfrak{n}_{4}$ & $\begin{array}{l}\omega=e^{1} \wedge e^{3}+e^{2} \wedge e^{4} \\
\theta=e^{2}\end{array}$ & \\
\hline $\mathfrak{r}_{3, \lambda} \times \mathbb{R}$ & $\begin{array}{l}\omega=2 e^{1} \wedge e^{2}+(1+\lambda) e^{1} \wedge e^{3}- \\
e^{2} \wedge e^{4}-e^{3} \wedge e^{4}\end{array}$ & $\omega=e^{1} \wedge e^{4}-e^{2} \wedge e^{3}$ \\
\hline$(\lambda \neq-1)$ & $\theta=e^{1}+e^{4}$ & $\theta=-(1+\lambda) e^{1}$ \\
\hline $\mathfrak{r}_{3,-1} \times \mathbb{R}$ & $\begin{array}{l}\omega=2 e^{1} \wedge e^{2}-e^{2} \wedge e^{4}-e^{3} \wedge e^{4} \\
\theta=e^{1}+e^{4}\end{array}$ & $\begin{array}{l}\omega=e^{1} \wedge e^{2}-e^{3} \wedge e^{4} \\
\theta=e^{1}\end{array}$ \\
\hline $\begin{array}{l}\mathfrak{r}_{4, \mu, \lambda} \\
(\lambda \neq-1)\end{array}$ & & $\begin{array}{l}\omega=e^{1} \wedge e^{3}+e^{2} \wedge e^{4} \\
\theta=-(\lambda+1) e^{1}\end{array}$ \\
\hline $\begin{array}{l}\mathfrak{r}_{4, \mu,-1} \\
(\mu \neq 1)\end{array}$ & & $\begin{array}{l}\omega=e^{1} \wedge e^{2}+e^{3} \wedge e^{4} \\
\theta=(1-\mu) e^{1},\end{array}$ \\
\hline $\mathfrak{r}_{4,1,-1}$ & & $\begin{array}{l}\omega=e^{1} \wedge e^{4}+e^{2} \wedge e^{3} \\
\theta=-2 e^{1}\end{array}$ \\
\hline $\mathfrak{r}_{3} \times \mathbb{R}$ & $\begin{array}{l}\omega=e^{1} \wedge e^{2}+2 e^{1} \wedge e^{3}-e^{2} \wedge \\
e^{4}-e^{3} \wedge e^{4}\end{array}$ & $\omega=e^{1} \wedge e^{4}-e^{2} \wedge e^{3}$ \\
\hline $\begin{array}{l}\mathfrak{r}_{4, \lambda} \\
(\lambda \neq 0)\end{array}$ & $\theta=e^{4}$ & $\begin{array}{l}\theta=-2 e^{1} \\
\omega=e^{1} \wedge e^{2}-e^{3} \wedge e^{4} \\
\theta=-2 \lambda e^{1}\end{array}$ \\
\hline $\mathfrak{r}_{4,0}$ & $\begin{array}{l}\omega=-e^{1} \wedge e^{2}+e^{2} \wedge e^{4}+e^{3} \wedge e^{4} \\
\theta=e^{4}\end{array}$ & $\begin{array}{l}e^{1} \wedge e^{3}+e^{2} \wedge e^{4} \\
\theta=-e^{1}\end{array}$ \\
\hline $\mathfrak{r}_{4}$ & & $\begin{array}{l}\omega=e^{1} \wedge e^{2}+e^{3} \wedge e^{4} \\
\theta=-2 e^{1}\end{array}$ \\
\hline $\mathfrak{r}_{3, \lambda}^{\prime} \times \mathbb{R}$ & $\omega=-e^{1} \wedge e^{2}+2 \lambda e^{1} \wedge e^{3}-e^{3} \wedge e^{4}$ & $\omega=e^{1} \wedge e^{4}-e^{2} \wedge e^{3}$ \\
\hline$(\lambda \neq 0)$ & $\theta=\lambda e^{1}+e^{4}$ & $\theta=-2 \lambda e^{1}$ \\
\hline $\mathfrak{r}_{3,0}^{\prime} \times \mathbb{R}$ & $\begin{array}{l}\omega=e^{1} \wedge e^{2}+e^{3} \wedge e^{4} \\
\theta=e^{4}\end{array}$ & \\
\hline $\begin{array}{l}\mathfrak{r}_{4, \mu, \lambda}^{\prime} \\
(\lambda \neq 0)\end{array}$ & & $\begin{array}{l}\omega=e^{1} \wedge e^{2}+e^{3} \wedge e^{4} \\
\theta=-2 \lambda e^{1}\end{array}$ \\
\hline
\end{tabular}

Let us show the last statement, i.e. the almost abelian Lie algebra $\mathfrak{r}_{3,0}^{\prime} \times \mathbb{R}$ admits LCS structures only of the first kind. Indeed, let us suppose that $\omega=$ $a_{1} f^{12}+a_{2} f^{13}+a_{3} f^{14}+a_{4} f^{23}+a_{5} f^{24}+a_{6} f^{34}, \theta=b_{1} f^{1}+b_{2} f^{2}+b_{3} f^{3}+b_{4} f^{4}$ is an LCS structure on this Lie algebra with $a_{i}, b_{j} \in \mathbb{R}$. From $d \omega=\theta \wedge \omega$ we obtain that $b_{2} \neq 0$. On the other hand, $f_{2} \in \mathfrak{z}(\mathfrak{g})$, then $\mathrm{L}_{f_{2}} \omega=0$. Thus $f_{2} \in \mathfrak{g}_{\omega}$ and $\theta\left(f_{2}\right)=b_{2} \neq 0$. Therefore $(\theta, \omega)$ is of the first kind.

We can summarize these results in Table 1, where we exhibit LCS structures of the first or second kind for each Lie algebra. The empty spaces mean that the corresponding Lie algebra does not admit any LCS structure of that specific kind. 


\subsection{Lattices in the associated LCS Lie groups}

4.2.1. Lattices in dimension 4 In this section we will determine up to Lie algebra isomorphism the almost abelian Lie algebras in Theorem 4.5 whose associated simply connected Lie groups admit lattices. In order to do this we use the classification of unimodular completely solvable Lie groups of type $\mathbb{R} \ltimes \mathbb{R}^{3}$ in [28] and Proposition 2.3.

Theorem 4.8. Let $G$ be a simply connected 4-dimensional unimodular almost abelian Lie group with a left invariant LCS structure, and let $\mathfrak{g}$ denote its Lie algebra. If $G$ admits lattices then $\mathfrak{g}$ is isomorphic to one of the following Lie algebras: $\mathfrak{h}_{3} \times \mathbb{R}, \mathfrak{n}_{4}, \mathfrak{r}_{3,-1} \times \mathbb{R}, \mathfrak{r}_{3,0}^{\prime} \times \mathbb{R}, \mathfrak{r}_{4, \lambda,-(1+\lambda)}$ for countably many values of $\lambda>1$, or $\mathfrak{r}_{4, \lambda,-\lambda / 2}^{\prime}$ for countably many values of $\lambda>0$.

Remark 4.9. It is easy to verify that if $\lambda^{\prime} \in\left\{\lambda,-(1+\lambda), \frac{1}{\lambda},-\frac{1}{1+\lambda},-\frac{\lambda}{1+\lambda},-\frac{\lambda+1}{\lambda}\right\}$

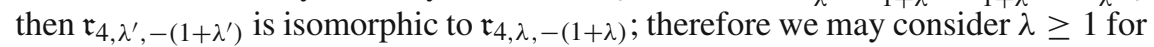
this family. In the same way, it is readily checked that $\mathfrak{r}_{4, \lambda^{\prime},-\lambda^{\prime} / 2}^{\prime}$ is isomorphic to $\mathfrak{r}_{4, \lambda,-\lambda / 2}^{\prime}$ if $\lambda^{\prime}= \pm \lambda$, so that in this case we may assume $\lambda>0$.

Proof. Let us consider the unimodular Lie algebras in Theorem 4.5, see Remark 4.6(ii).

In the nilpotent case, the simply connected nilpotent Lie groups corresponding to the Lie algebras $\mathfrak{h}_{3} \times \mathbb{R}$ and $\mathfrak{n}_{4}$ admit lattices due to Malcev's criterion, since these Lie algebras have rational structure constants for some basis.

Next, we consider the case of the completely solvable non-nilpotent Lie algebras $\mathfrak{r}_{3,-1} \times \mathbb{R}, \mathfrak{r}_{4, \lambda,-(1+\lambda)}(\lambda \geq 1)$ and $\mathfrak{r}_{4,-1 / 2}$. It is well known that the simply connected Lie group $\mathrm{Sol}^{3}$ corresponding to $\mathfrak{r}_{3,-1} \simeq \mathfrak{s o l}^{3}$ admits lattices (see for instance [30]), and therefore $\mathrm{Sol}^{3} \times \mathbb{R}$ admits lattices as well. Now we use the classification given in [28], noting that $\mathfrak{r}_{4, \lambda,-(1+\lambda)} \simeq \mathfrak{s o l}_{\lambda}^{4}(\lambda>1), \mathfrak{r}_{4,1,-2} \simeq \mathfrak{s o r}_{0}^{4}$ and $\mathfrak{r}_{4,-1 / 2} \simeq \mathfrak{s o l}_{0}^{\prime 4}$. The simply connected Lie group associated to $\mathfrak{s o l}_{\lambda}^{4}$ admits lattices for countably many $\lambda^{\prime} s$ (see [28, Proposition 2.1]), whereas the Lie groups $\mathrm{Sol}_{0}^{4}$ and $\mathrm{Sol}_{0}^{\prime 4}$ associated to $\mathfrak{s o l}_{0}^{4}$ and $\mathfrak{s o l}_{0}^{\prime 4}$ do not admit any lattices [28, Proposition 2.2]. Note that $\mathrm{Sol}_{0}^{4}=G_{0}$ from 3.2.2.

Finally, we take into account the non-completely solvable Lie algebras $\mathfrak{r}_{3,0}^{\prime} \times \mathbb{R}$ and $\mathfrak{r}_{4, \lambda,-\lambda / 2}^{\prime}(\lambda>0)$. It is well known that the simply connected Lie group $E(2)$ corresponding to $\mathfrak{r}_{3,0}^{\prime} \simeq \mathfrak{e}(2)$ admits lattices (this fact is also an easy application of Proposition 2.3), and therefore $E(2) \times \mathbb{R}$ admits lattices, too. The Lie algebra $\mathfrak{r}_{4, \lambda,-\lambda / 2}^{\prime}(\lambda>0)$ is isomorphic to $\mathfrak{g}_{b}$ from 3.2 .2 , for $b=\frac{1}{\lambda}$, and it has already been proved in Theorem 3.9 that the corresponding simply connected Lie groups $G_{b}$ admit lattices for countably many values of the parameter $b>0$.

Remark 4.10. The Lie algebras $\mathfrak{n}_{4}, \mathfrak{r}_{3,-1} \times \mathbb{R}, \mathfrak{r}_{4, \lambda,-(1+\lambda)}$ for countably many values of $\lambda>1$ and $\mathfrak{r}_{3,0}^{\prime} \times \mathbb{R}$ provide examples of solvmanifolds with an LCS structure which carry no LCK structure (coming from a left invariant LCK structure on the Lie group). We point out that in [6] it was shown that a nilmanifold associated to $\mathfrak{n}_{4}$ admits an LCS structure of the first kind but does not admit any LCK metric. Moreover, this nilmanifold is not the product of a 3-dimensional compact manifold and a circle. 
4.2.2. Lattices in dimension $\geq 6$ In this subsection we will provide examples of almost abelian solvmanifolds with an LCS structure. For $n \geq 2$, let $\mathfrak{g}$ be the $(2 n+2)$ dimensional unimodular almost abelian Lie algebra given by $\mathfrak{g}=\mathbb{R} f_{1} \ltimes_{M} \mathbb{R}^{2 n+1}$ with

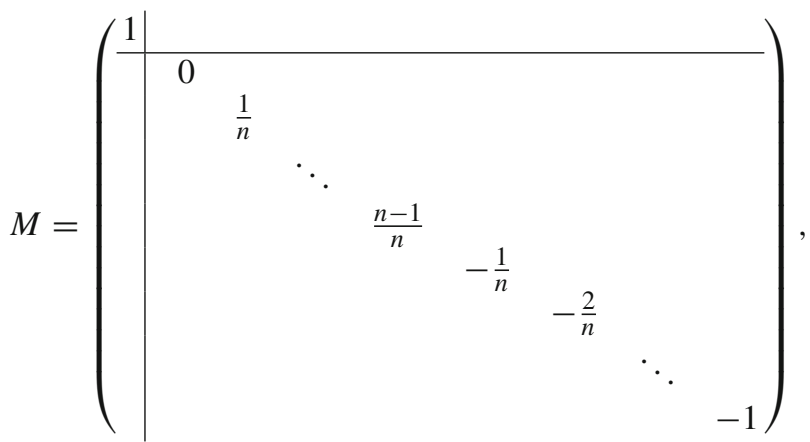

in a basis $\left\{f_{2}, u_{1}, \ldots, u_{n}, v_{1}, \ldots, v_{n}\right\}$ of $\mathbb{R}^{2 n+1}$. Note that $M$ is given by (21) with

$$
\begin{aligned}
& \mu=1, \quad \lambda=-\frac{1}{2 n}, \quad w=0, \\
& B=\operatorname{diag}\left(\frac{1}{2 n}, \frac{3}{2 n}, \ldots, \frac{2 n-1}{2 n},-\frac{1}{2 n},-\frac{3}{2 n}, \ldots,-\frac{2 n-1}{2 n}\right) \in \mathfrak{s p}(n, \mathbb{R}) .
\end{aligned}
$$

According to Theorem 4.1 the Lie algebra $\mathfrak{g}$ admits an LCS form $\omega=f^{1} \wedge f^{2}+$ $\sum_{i=1}^{n} u^{i} \wedge v^{i}$ with Lee form $\theta=\frac{1}{n} f^{1}$. From Corollary 4.3 we have that this LCS structure is of the second kind.

The simply connected Lie group associated to $\mathfrak{g}$ is $G=\mathbb{R} \ltimes_{\phi} \mathbb{R}^{2 n+1}$ where $\phi$ is given by

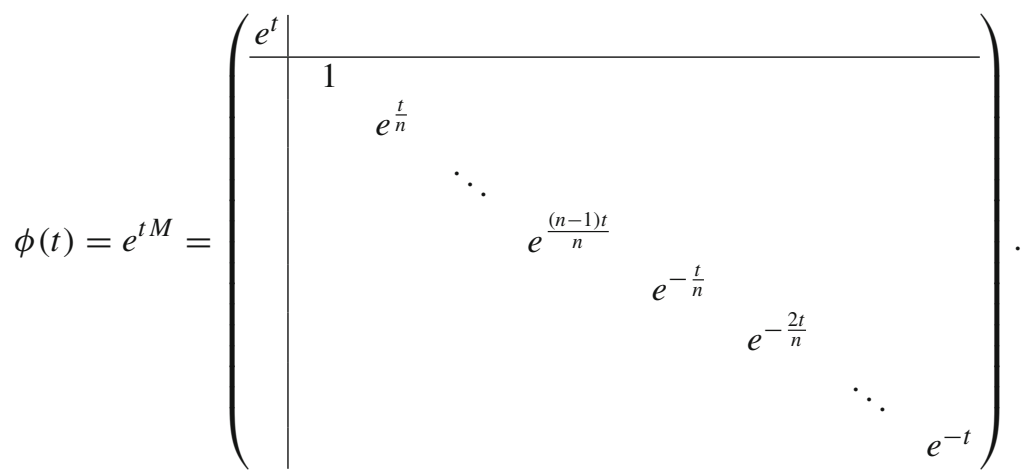

The characteristic polynomial of $\phi(t)$ is $p(x)=(x-1)\left(x-\rho^{2}\right)\left(x-\rho^{-2}\right)\left(x-\rho^{4}\right)\left(x-\rho^{-4}\right) \ldots\left(x-\rho^{2 n}\right)\left(x-\rho^{-2 n}\right)$, where $\rho=e^{\frac{t}{2 n}}$. Fixed $m \in \mathbb{N}, m>2$, we define

$$
t_{m}=n \operatorname{arccosh}\left(\frac{m}{2}\right), \quad t_{m}>0 .
$$


Then $\rho_{m}=e^{\frac{t_{m}}{2 n}}$ satisfies $\rho_{m}^{2}+\rho_{m}^{-2}=m$.

We define the following real sequence $a_{0}=2, a_{1}=m, a_{k}=\rho_{m}^{2 k}+\rho_{m}^{-2 k}$ for $k=$ $3, \ldots, n$. It is easy to see that $a_{k}$ satisfies $a_{k+1}=m a_{k}-a_{k-1}$ and therefore $a_{k} \in \mathbb{Z}$ for all $k$. As a consequence we have that $\left(x-\rho_{m}^{2 k}\right)\left(x-\rho_{m}^{-2 k}\right)=x^{2}-a_{k} x+1 \in \mathbb{Z}[x]$, thus we can write

$$
p(x)=x^{2 n+1}-m_{2 n} x^{2 n}+m_{2 n-1} x^{2 n-1}+\cdots+m_{1} x-1
$$

for some $m_{1}, m_{2}, \ldots, m_{2 n} \in \mathbb{Z}$. Since all the roots of $p$ are different, we have that $\phi\left(t_{m}\right)$ is conjugated to the companion matrix $B$ of $p$, that is,

$$
B_{m}=\left(\begin{array}{ccccc}
0 & & & & 1 \\
1 & 0 & & & -m_{1} \\
& \ddots & \ddots & & \vdots \\
& & 1 & 0 & -m_{2 n-1} \\
& & & 1 & m_{2 n}
\end{array}\right) .
$$

According to Proposition 2.3, the group $G$ admits lattices. One such lattice is given by

$$
\Gamma_{m}:=t_{m} \mathbb{Z} \ltimes_{\phi} P_{m}^{-1} \mathbb{Z}^{2 n+1},
$$

for each $m>2$, where $P_{m}$ satisfies $P_{m} \phi\left(t_{m}\right) P_{m}^{-1}=B_{m}$. Therefore for all $m>2$, $M_{m}:=\Gamma_{m} \backslash G$ is a solvmanifold with an LCS structure.

Proposition 4.11. With notation as above, the solvmanifolds $M_{m}$ with $m \in \mathbb{N}, m>$ 2, are pairwise non homeomorphic.

Proof. Let us assume that $M_{r}$ and $M_{s}$, with $r, s>2$ are homeomorphic. Then $\pi_{1}\left(M_{r}\right)$ is isomorphic to $\pi_{1}\left(M_{S}\right)$. Since $G$ is simply connected, we have that the fundamental groups of these solvmanifolds are isomorphic to the lattices $\Gamma_{r}$ and $\Gamma_{s}$, so that these lattices are isomorphic. Since $G$ is completely solvable, the Saito's rigidity theorem [37] implies that this isomorphism extends to a Lie group automorphism of $G$. Since the lattices differ by an automorphism of $G$, it follows from [20, Theorem 2.5] that the integer matrix $B_{r}$ [as in (22)] is conjugated either to $B_{s}$ or to its inverse in $G L(n, \mathbb{Z})$. It can be seen that this happens if and only if $r=s$.

Remark 4.12. It is easy to see that the LCS solvmanifolds $M_{m}$ do not admit invariant complex structures. However, they do admit invariant symplectic structures, for instance, $\eta=f^{1} \wedge u^{1}+f^{2} \wedge v^{n}+\sum_{i=1}^{n-1} u^{i+1} \wedge v^{i}$.

4.2.3. De Rham and adapted cohomology of the LCS solvmanifolds $\Gamma_{m} \backslash G \quad$ We begin with the study of the de Rham cohomology of the solvmanifold $M_{m}=\Gamma_{m} \backslash G$. Since $G$ is completely solvable, it follows from (8) that $H_{d R}^{k}\left(M_{m}\right)$ is isomorphic to the Lie algebra cohomology group $H^{k}(\mathfrak{g})$. Therefore, we need to study the Chevalley-Eilenberg complex of the almost abelian Lie algebra $\mathfrak{g}=\mathbb{R} f_{1} \ltimes_{M} \mathbb{R}^{2 n+1}$ with $M$ as in (21). After a change of basis in the ideal $\mathfrak{u}=\mathbb{R}^{2 n+1}$, we can assume that $M$ is given by 


$$
M=\operatorname{diag}\left(0, \frac{1}{n}, \frac{2}{n}, \ldots, 1,-\frac{1}{n},-\frac{2}{n}, \ldots,-1\right) .
$$

According to [38], the $k^{t h}$ Betti number of $\mathfrak{g}, \beta_{k}^{n}=\operatorname{dim} H^{k}(\mathfrak{g})$, can be computed as follows, where $Z^{j}(\mathfrak{g})=\left\{\alpha \in \bigwedge^{j} \mathfrak{g}^{*}: d \alpha=0\right\}$ :

$$
\beta_{k}^{n}=\operatorname{dim} H^{k}(\mathfrak{g})=\operatorname{dim} Z^{k}(\mathfrak{g})+\operatorname{dim} Z^{k-1}(\mathfrak{g})-\left(\begin{array}{c}
2 n+2 \\
k-1
\end{array}\right) .
$$

In order to compute $\operatorname{dim} Z^{k}(\mathfrak{g})$, note that we can decompose the ideal $\mathfrak{u}$ as $\mathfrak{u}=\mathbb{R} f_{2} \oplus \mathfrak{g}^{\prime}$, where $f_{2}$ is a generator of the center of $\mathfrak{g}$. Let us denote for simplicity $\mathfrak{v}:=\mathfrak{g}^{\prime}$.

Recalling that $\beta_{1}^{n}=\operatorname{dim}(\mathfrak{g} /[\mathfrak{g}, \mathfrak{g}])$, it follows that $\beta_{1}^{n}=2$. Indeed, a basis of $Z^{1}(\mathfrak{g})$ is given by $\left\{f^{1}, f^{2}\right\}$. Due to Poincaré duality, we get that $\beta_{2 n+1}^{n}=\beta_{1}^{n}=2$. Clearly, $\beta_{0}^{n}=\beta_{2 n+2}^{n}=1$.

Let us consider now $2 \leq k \leq 2 n$. Given a closed $k$-form $\alpha \in Z^{k}(\mathfrak{g})$, it can be decomposed uniquely as $\alpha=f^{1} \wedge \beta+f^{2} \wedge \gamma+\delta$, with $\beta \in \wedge^{k-1} \mathfrak{u}^{*}$, $\gamma \in \bigwedge^{k-1} \mathfrak{v}^{*}, \delta \in \bigwedge^{k} \mathfrak{v}^{*}$, with $d \gamma=0$ and $d \delta=0$. Therefore, we have a one to one correspondence

$$
Z^{k}(\mathfrak{g}) \longleftrightarrow\left(\bigwedge^{k-1} \mathfrak{u}^{*}\right) \times\left(\bigwedge^{k-1} \mathfrak{v}^{*} \cap Z^{k-1}(\mathfrak{g})\right) \times\left(\bigwedge^{k} \mathfrak{v}^{*} \cap Z^{k}(\mathfrak{g})\right)
$$

In order to compute $\operatorname{dim}\left(\bigwedge^{k} \mathfrak{v}^{*} \cap Z^{k}(\mathfrak{g})\right)$, we consider, for $1 \leq p, q \leq n$, the set

$$
\left\{\left(i_{1}, \ldots, i_{p}, j_{1}, \ldots, j_{q}\right): 1 \leq i_{1}<\cdots<i_{p} \leq n, 1 \leq j_{1}<\cdots<j_{q} \leq n, \sum_{r=1}^{p} i_{r}=\sum_{s=1}^{q} j_{s}\right\},
$$

and let us denote by $d_{p, q}^{n}$ its cardinal. Setting $D_{k}^{n}:=\sum_{p+q=k} d_{p, q}^{n}$ for $k \geq 2$, it can be shown that $\operatorname{dim}\left(\bigwedge^{k} \mathfrak{v}^{*} \cap Z^{k}(\mathfrak{g})\right)=D_{k}^{n}$. Let us also define $d_{0,0}^{n}=1, d_{1,0}^{n}=d_{0,1}^{n}=0$, so that $D_{0}^{n}=1, D_{1}^{n}=0$. Using this together with (23), we readily obtain that

$$
\beta_{k}^{n}=D_{k-2}^{n}+2 D_{k-1}^{n}+D_{k}^{n}, \quad k \geq 2 .
$$

Even though we do not obtain an explicit formula for the Betti numbers of $M_{m}$, using (24) we are able to obtain some properties concerning the parity of these numbers.

It is easy to see that $d_{p, q}^{n}=d_{q, p}^{n}$ and $d_{p, q}^{n}=d_{n-p, n-q}^{n}$. We can summarize some properties about $d_{p, q}^{n}$ and $D_{k}^{n}$ in the following lemma.

Lemma 4.13. (i) $d_{1,1}^{n}=d_{n-1, n-1}^{n}=n$ and $d_{k, k}^{n} \equiv\left(\begin{array}{l}n \\ k\end{array}\right)(\bmod 2)$.

(ii) If $n$ is even then $d_{1,2}^{n}=\frac{n(n-2)}{4}$ and if $n$ is odd then $d_{1,2}^{n}=\left(\frac{n-1}{2}\right)^{2}$.

(iii) $D_{k}^{n}=D_{2 n-k}^{n}$ for any $k=0, \ldots, n$, with $D_{0}^{n}=1, D_{1}^{n}=0, D_{2}^{n}=n$.

(iv) If $k$ is odd, then $D_{k}^{n}$ is even.

(v) $D_{2 k}^{n} \equiv\left(\begin{array}{l}n \\ k\end{array}\right)(\bmod 2)$.

Taking this lemma into account, the proof of the next result is straightforward. 
Table 2. Betti numbers

\begin{tabular}{lll}
\hline$n$ & $\operatorname{dim} \mathfrak{g}$ & Betti numbers \\
\hline 2 & 6 & $(1,2,3,4,3,2,1)$ \\
3 & 8 & $(1,2,4,8,10,8,4,2,1)$ \\
4 & 10 & $(1,2,5,12,20,24,20,12,5,2,1)$ \\
5 & 12 & $(1,2,6,18,37,56,64,56,37,18,6,2,1)$ \\
6 & 14 & $(1,2,7,24,61,116,167,188,167,116,61,24,7,2,1)$ \\
\hline
\end{tabular}

Proposition 4.14. (i) $\beta_{0}^{n}=1, \beta_{1}^{n}=2$ and $\beta_{2}^{n}=n+1$.

(ii) If $n$ is even, then $\beta_{3}^{n}=\frac{n(n+1)}{2}$ and if $n$ is odd then $\beta_{3}^{n}=\frac{(n+1)^{2}}{2}$.

(iii) If $k$ is odd, then $\beta_{k}^{n}$ is even.

(iv) $\beta_{2 k}^{n} \equiv\left(\begin{array}{c}n+1 \\ k\end{array}\right)(\bmod 2)$.

Remark 4.15. According to Lucas' Theorem, the parity of the binomial coefficient $\left(\begin{array}{l}r \\ s\end{array}\right)$ can be determined from the binary representation of $r$ and $s$. Indeed, if $r=$ $\sum_{i=1}^{m} a_{i} 2^{i}$ and $s=\sum_{i=1}^{m} b_{i} 2^{i}$ with $a_{i}, b_{i} \in\{0,1\}, a_{m} \neq 0$, then

$$
\left(\begin{array}{l}
r \\
s
\end{array}\right) \equiv \prod_{i=1}^{m}\left(\begin{array}{l}
a_{i} \\
b_{i}
\end{array}\right)(\bmod 2)
$$

where $\left(\begin{array}{l}a_{i} \\ b_{i}\end{array}\right)=0$ if $a_{i}<b_{i}$. In particular, $\left(\begin{array}{l}r \\ s\end{array}\right)$ is even if and only there exists $i$ such that $a_{i}=0$ and $b_{i}=1$. For instance, if $r=2^{l}-1$ then $\left(\begin{array}{l}r \\ s\end{array}\right)$ is odd for any $0 \leq s \leq r$, and if $r=2^{l}$ then $\left(\begin{array}{l}r \\ s\end{array}\right)$ is even for any $1 \leq s \leq r-1$.

We exhibit in Table 2 the Betti numbers in low dimensions. For instance, in the 6-dimensional case we obtain $\beta_{0}=\beta_{6}=1, \beta_{1}=\beta_{5}=2, \beta_{2}=\beta_{4}=3, \beta_{3}=4$.

Next, we will study the adapted cohomology of the solvmanifold $M_{m}=\Gamma_{m} \backslash G$. Since $G$ is completely solvable, it follows from (9) that $H_{\theta}^{k}\left(M_{m}\right)$ is isomorphic to the Lie algebra adapted cohomology group $H_{\theta}^{k}(\mathfrak{g})$. We will work in the same way as in the de Rham cohomology case.

Let $\tilde{\beta}_{k}^{n}=\operatorname{dim} H_{\theta}^{k}(\mathfrak{g})$ be the $k^{t h}$ adapted Betti number of $\mathfrak{g}$. These numbers satisfy an equation as (23). Indeed, setting $Z_{\theta}^{j}(\mathfrak{g})=\left\{\alpha \in \bigwedge^{j} \mathfrak{g}^{*}: d_{\theta} \alpha=0\right\}$ we have

$$
\tilde{\beta}_{k}^{n}=\operatorname{dim} H_{\theta}^{k}(\mathfrak{g})=\operatorname{dim} Z_{\theta}^{k}(\mathfrak{g})+\operatorname{dim} Z_{\theta}^{k-1}(\mathfrak{g})-\left(\begin{array}{c}
2 n+2 \\
k-1
\end{array}\right) .
$$

Note that $\operatorname{dim} Z_{\theta}^{1}(\mathfrak{g})=2$. Let us denote by $\tilde{f}^{1}$ and $\tilde{f}^{2}$ the generators of $Z_{\theta}^{1}(\mathfrak{g})$ where $\tilde{f}^{1}$ coincides with $f^{1}$ from above. Then we can decompose the ideal $\mathfrak{u}$ as $\mathfrak{u}=\mathbb{R} \tilde{f}_{2} \oplus \mathfrak{v}$ for some $2 n$-dimensional abelian subalgebra $\mathfrak{v}$. It is easy to see that

$$
\tilde{\beta}_{1}^{n}=1, \quad \tilde{\beta}_{2}^{n}=n+1 .
$$

Let us consider now $2 \leq k \leq 2 n$. Given a $d_{\theta}$-closed $k$-form $\alpha \in Z_{\theta}^{k}(\mathfrak{g})$, it can be decomposed uniquely as $\alpha=\tilde{f}^{1} \wedge \beta+\tilde{f}^{2} \wedge \gamma+\delta$, with $\beta \in \bigwedge^{k-1} \mathfrak{u}^{*}$, 
Table 3. Adapted Betti numbers

\begin{tabular}{lll}
\hline$n$ & $\operatorname{dim} \mathfrak{g}$ & adapted Betti numbers \\
\hline 2 & 6 & $(0,1,3,4,3,1,0)$ \\
3 & 8 & $(0,1,4,7,8,7,4,1,0)$ \\
4 & 10 & $(0,1,5,12,19,22,19,12,5,1,0)$ \\
5 & 12 & $(0,1,6,17,35,55,63,55,35,17,6,1,0)$ \\
6 & 14 & $(0,1,7,24,59,112,165,188,165,112,59,24,7,1,0)$ \\
\hline
\end{tabular}

$\gamma \in \bigwedge^{k-1} \mathfrak{v}^{*}, \delta \in \bigwedge^{k} \mathfrak{v}^{*}$, with $d_{\theta} \gamma=0$ and $d_{\theta} \delta=0$. Therefore, we have a one to one correspondence

$$
Z_{\theta}^{k}(\mathfrak{g}) \longleftrightarrow\left(\bigwedge^{k-1} \mathfrak{u}^{*}\right) \times\left(\bigwedge^{k-1} \mathfrak{v}^{*} \cap Z_{\theta}^{k-1}(\mathfrak{g})\right) \times\left(\bigwedge^{k} \mathfrak{v}^{*} \cap Z_{\theta}^{k}(\mathfrak{g})\right) .
$$

In order to compute $\operatorname{dim}\left(\bigwedge^{k} \mathfrak{v}^{*} \cap Z_{\theta}^{k}(\mathfrak{g})\right)$, we consider, for $1 \leq p, q \leq n$, the set

$$
\left\{\left(i_{1}, \ldots, i_{p}, j_{1}, \ldots, j_{q}\right): 1 \leq i_{1}<\cdots<i_{p} \leq n, 1 \leq j_{1}<\cdots<j_{q} \leq n, \sum_{r=1}^{p} i_{r}+1=\sum_{s=1}^{q} j_{s}\right\},
$$

and let us denote by $\tilde{d}_{p, q}^{n}$ its cardinal. Setting $\tilde{D}_{k}^{n}:=\sum_{p+q=k} \tilde{d}_{p, q}^{n}$ for $k \geq 2$, it can be shown that $\operatorname{dim}\left(\bigwedge^{k} \mathfrak{v}^{*} \cap Z_{\theta}^{k}(\mathfrak{g})\right)=\tilde{D}_{k}^{n}$. Let us also define $\tilde{d}_{0,0}^{n}=\tilde{d}_{1,0}^{n}=0$, $\tilde{d}_{0,1}^{n}=1$, so that $\tilde{D}_{0}^{n}=0, \tilde{D}_{1}^{n}=1$.

From (25), we readily obtain that

$$
\tilde{\beta}_{k}^{n}=\tilde{D}_{k-2}^{n}+2 \tilde{D}_{k-1}^{n}+\tilde{D}_{k}^{n}, \quad k \geq 2 .
$$

It is easy to see that $\tilde{d}_{p, q}^{n}=\tilde{d}_{n-q, n-p}^{n}$. Then we have that $\tilde{D}_{k}^{n}=\tilde{D}_{2 n-k}^{n}$ and therefore

$$
\tilde{\beta}_{k}^{n}=\tilde{\beta}_{2 n-k}^{n}, \quad \sum_{k=0}^{2 n+2}(-1)^{k} \tilde{\beta}_{k}^{n}=0 .
$$

Using (26) and these facts we can compute the adapted Betti numbers in some low-dimensional cases, which are shown in Table 3.

Acknowledgements. We would like to thank A. Moroianu for his help with integer polynomials, I. Dotti for useful comments and suggestions and the anonymous referee for helpful observations. The authors were partially supported by CONICET, ANPCyT and SECyTUNC (Argentina).

\section{References}

[1] Alekseevsky, D., Cortés, V., Hasegawa, K., Kamishima, Y.: Homogeneous locally conformally Kähler and Sasaki manifolds. Intern. J. Math. 26, 1541001 (2015)

[2] Andrada, A., Barberis, M.L., Dotti, I.G., Ovando, G.P.: Product structures on four dimensional solvable Lie algebras. Homol. Homotopy Appl. 7, 9-37 (2005) 
[3] Andrada, A., Origlia, M.: Locally conformally Kähler structures on unimodular Lie groups. Geom. Dedicata 179, 197-216 (2015)

[4] Andriot, D., Goi, E., Minasian, R., Petrini, M.: Supersymmetry breaking branes on solvmanifolds and de Sitter vacua in string theory. J. High Energ. Phys. 2011(5), 28 (2011). doi:10.1007/JHEP05(2011)028

[5] Banyaga, A.: Examples of non $d_{\theta}$-exact locally conformal symplectic forms. J. Geom. 87, 1-13 (2007)

[6] Bazzoni, G., Marrero, J.C.: On locally conformal symplectic manifolds of the first kind, to appear in Bulletin de Sciences Mathématiques. arXiv:1510.04947

[7] Bock, C.: On low-dimensional solvmanifolds. Asian J. Math. 20, 199-262 (2016)

[8] Borwein, P., Hare, K.: Non-trivial quadratic approximations to zero of a family of cubic Pisot numbers. Am. Math. Soc. 355, 4767-4779 (2003)

[9] Console, S., Macri, M.: Lattices, cohomology and models of six dimensional almost abelian solvmanifolds, preprint 2012. arXiv:1206.5977v1

[10] Cordero, L.A., Fernández, M., de Léon, M.: Compact locally conformal Kähler nilmanifolds. Geom. Dedicata 21, 187-192 (1986)

[11] Dragomir, S., Ornea, L.: Locally Conformal Kähler Geometry. Progress in Mathematics 155. Birkhäuser, Basel (1998)

[12] Gauduchon, P., Moroianu, A., Ornea, L.: Compact homogeneous lcK manifolds are Vaisman. Math. Ann. 361, 1043-1048 (2015)

[13] Gordon, C., Wilson, E.: The spectrum of the Laplacian on Riemannian Heisenberg manifolds. Mich. Math. J. 33, 253-271 (1986)

[14] Gray, A., Hervella, L.: The sixteen classes of almost Hermitian manifolds and their linear invariants. Ann. Mat. Pura Appl. 123, 35-58 (1980)

[15] Guedira, F., Lichnerowicz, A.: Géométrie des algèbres de Lie locales de Kirillov. J. Math. Pures Appl. 63, 407-484 (1984)

[16] Haller, S.: Some properties of locally conformal symplectic manifolds. In: Infinite Dimensional Lie Groups in Geometry and Representation Theory, pp 92-104. World Scientific Publishing Company, River Edge, NJ (2002)

[17] Haller, S., Rybicki, T.: Reduction for locally conformal symplectic manifolds. J. Geom. Phys. 37, 262-271 (2001)

[18] Hasegawa, K.: Complex and Kähler structures on compact solvmanifolds. J. Symplectic Geom. 3, 749-767 (2005)

[19] Hattori, A.: Spectral sequence in the de Rham cohomology of fibre bundles. J. Fac. Sci. Univ. Tokyo Sect. I 8, 289-331 (1960)

[20] Huang, H.: Lattices and harmonic analysis on some 2-step solvable Lie groups. J. Lie Theory 13, 77-89 (2003)

[21] Kamishima, Y.: Note on locally conformal Kähler surfaces. Geom. Dedicata 84, 115124 (2001)

[22] Kasuya, H.: Vaisman metrics on solvmanifolds and Oeljeklaus-Toma manifolds. Bull. Lond. Math. Soc. 45, 15-26 (2013)

[23] Kronecker, L.: Zwei Sätze über Gleichungen mit ganzzahligen Coefficienten. J. Reine Angew. Math. 53, 173-175 (1857)

[24] Lauret, J., Rodríguez Valencia, E.: On the Chern-Ricci flow and its solitons for Lie groups. Math. Nachr. 288, 1512-1526 (2015)

[25] Lauret, J., Will, C.: On the symplectic curvature flow for locally homogeneous manifolds, to appear in J. Symplectic Geom. arXiv:1405.6065

[26] Lê, H.V., Vanžura, J.: Cohomology theories on locally conformal symplectic manifolds. Asian J. Math. 19, 45-82 (2015) 
[27] Lee, H.C.: A kind of even dimensional differential geometry and its application to exterior calculus. Am. J. Math. 65, 433-438 (1943)

[28] Lee, J.B., Lee, K.B., Shin, J., Yi, S.: Unimodular groups of type $\mathbb{R}^{3} \rtimes \mathbb{R}$. J. Korean Math. Soc. 44, 1121-1137 (2007)

[29] Malcev, A.: On solvable Lie algebras. Bull. Acad. Sci. URSS. Sr. Math. [Izvestia Akad. Nauk SSSR] 9, 329-356 (1945)

[30] Medina, A., Revoy, P.: Lattices in symplectic Lie groups. J. Lie Theory 17, 27-39 (2007)

[31] Millionschikov, D.: Cohomology of solvable Lie algebras and solvmanifolds. Math. Notes 77, 61-71 (2005)

[32] Milnor, J.: Curvatures of left invariant metrics on Lie groups. Adv. Math. 21, 293-329 (1976)

[33] Moroianu, A., Ornea, L.: Transformations of locally conformally Kähler manifolds. Manuscr. Math. 130, 93-100 (2009)

[34] Ornea, L.: Locally conformal Kähler manifolds. A selection of results. Lect. Notes Semin. Interdiscip. Mat., IV, 121-152, S.I.M. Dep. Mat. Univ. Basilicata, Potenza (2005)

[35] Ornea, L., Verbitsky, M.: Locally conformal Kähler manifolds with potential. Math. Ann. 348, 25-33 (2010)

[36] Ovando, G.: Complex, symplectic and Kähler structures on four dimensional Lie groups. Rev. Unión Mat. Argent. 45, 55-68 (2004)

[37] Saito, M.: Sur certains groupes de Lie résolubles II. Sci. Papers Coll. Gen. Ed. Univ. Tokyo 7, 157-168 (1957)

[38] Santharoubane, L.J.: Cohomology of Heisenberg Lie algebras. Proc. Am. Math. Soc. 87, 23-28 (1983)

[39] Sawai, H.: Locally conformal Kähler structures on compact nilmanifold with leftinvariant complex structures. Geom. Dedicata 125, 93-101 (2007)

[40] Sawai, H.: Locally conformal Kähler structures on compact solvmanifolds. Osaka J. Math. 49, 1087-1102 (2012)

[41] Tricerri, F.: Some examples of locally conformal Kähler manifolds. Rend. Sem. Mat. Univ. Politec. Torino 40, 81-92 (1982)

[42] Vaisman, I.: On locally conformal almost Kähler manifolds. Isr. J. Math. 24, 338-351 (1976)

[43] Vaisman, I.: Locally conformal symplectic manifolds. Internat. J. Math. Sci. 12, 521$536(1985)$ 